\title{
LA CULTURA MUSIVA DI CAGLIARI
}

LUIGI QuaTTROCCHI

Universidad Carlos III

Recibido: 8/04/2015

Revisado: 9/05/2015
Aceptado: 9/05/2015

Publicado: 30/05/2015

\section{RIASSUNTO}

Dal 1981 a oggi la cultura musiva di Cagliari non è stata quasi mai studiata. In questi 30 anni lo studio della musivaria romana ha compiuto notevoli passi in avanti, per questa ragione sembra adatto apportare delle modifiche ai vecchi dati pubblicati nel 1981, revisionando 20 mosaici dell'antica città di Caralis, e aggiungendo alcune nuove scoperte. Con questa analisi si vede chiaramente che la cultura musiva calaritana si distacca molto dalla cultura dell'Isola di Sardegna, soprattutto perché nei vari secoli vede all'interno di questa città operare molti musivari differenti, con differenti background.

\section{ABSTRACT}

From 1981 to present day, culture mosaic of Cagliari was hardly studied. In these 30 years the study of Roman musivaria has made significant steps forward, for this reason it seems appropriate to make change to old data published in 1981, reviewing 20 mosaics of ancient city of Caralis, and adding some new discoveries. Thanks to this analysis it is clear the culture mosaic of Cagliari is detached from the culture of Sardinia, especially because in Cagliari operate many different musivari, with different background.

\section{KEYWORDS}

Musivaria; Mosaics; Sardinia; Cagliari; Opus Signinum. 
Le fonti antiche ci danno alcune notizie sulla vecchia Cagliari: secondo Solino sarebbe stata fondata dal mitico re tessalo Aristeo (Solin. IV 2), mentre Pausania (Pausan. X 17,9) ritiene che la fondazione sia ad opera dei Cartaginesi. Ovviamente anche Plinio fornisce citazioni su Caralis e i suoi abitanti (Plinio III 7,85), mentre Cesare ci da informazioni sulla fedeltà dei cittadini alla propria persona, durante la guerra civile (Caes., De Bell. Civ. I 30), che proprio in virtù di questo la elevò a municipium. Cagliari era iscritta alla tribù Quirina (CIL, X, 7587, 7598, 7599, 7603), e da non dimenticare sono le funzioni del porto di Cagliari, dei suoi naviculari et negotiantes (CIL, XIV, 4549), ricordate nel Piazzale delle Corporazioni a Ostia.

La città di Cagliari corrisponde all'antica città fenicio-punica di KRLY, e successivamente alla Karales o Caralis romana, e si trova alla base del versante occidentale di Capo Sant'Elia, un promontorio che si estende nella porzione mediana del Golfo degli Angeli, nella Sardegna meridionale. La città appare come un sito pluristratificato, dove le evidenze archeologiche del VII secolo a.C. si fondono con la città moderna. Non è facile capire quale fosse l'assetto della città in antico, anche perché ha avuto più nuclei insediativi, infatti una prima colonia fenicia e successivamente punica si trovava in una zona decentrata a nord-ovest rispetto all'attuale centro cittadino, in prossimità dello Stagno di Santa Gilla. Il tessuto romano si innesta dunque in un contesto preesistente, con una pianificazione delle risorse primarie già in essere, con i conseguenti collegamenti tra apparati abitativi e territoriali. Non di poco conto è la differente concezione della città tra le due diverse culture, quella punica da un lato e quella romana dall'altro: i Fenici ed i Punici avevano una concezione dello spazio strettamente correlata con l'ambiente, dunque anche la scelta dei luoghi di culto, che fossero complessi templari o tofet, era ponderata in base all'ambiente circostante; i Romani avevano una visione più pratica e politica della città e dei suoi spazi, e in buona parte anche economica, per questo reinterpretano edifici già esistenti, o ne costruiscono di nuovi in luoghi totalmente differenti rispetto al precedente insediamento. Così avviene a Caralis, dove il primo nucleo romano, il Foro, lo ritroviamo nella odierna Piazza del Carmine (Colavitti Deplano, 2002, 1119). In questo caso i Romani utilizzarono forme di archetipi monumentali già esistenti, come ad esempio il tempio-teatro di Via Malta, una tipologia monumentale attestata in ambiente medio-italico. Questo tipo di architettura che esula dal substrato preesistente si rispecchia anche nelle produzioni ceramiche, che vede un vasto uso di forme e tipologie tipiche del territorio centro-italico e in qualche modo etruschizzante. La Cagliari romana ha una forte zonizzazione funzionale, infatti basti pensare alla ripartizione degli edifici strettamente collegati al suo porto. Questo dualismo si riflette anche nelle diverse organizzazioni spaziali dell'agro cagliaritano. Il progressivo insediamento romano vede la creazione di un complesso termale adiacente al Foro, mentre l'edilizia abitativa si colloca sempre in prossimità del Foro, nel settore nord-occidentale. Sopra un promontorio nascerà il complesso residenziale noto come la villa di Tigellio, in asse con il sopraelevato Anfiteatro. Dell'attività produttiva ne abbiamo testimonianza grazia ad un impianto detto fullonica, in prossimità dell'area portuale. Si ha inoltre un dislocamento delle aree adibite a necropoli: nella parte occidentale ritroviamo la necropoli di Tuvixeddu, con un settore punico ed un settore romano, mentre ad oriente ritroviamo la necropoli di Bonaria. Lo schema stradale non è noto, anche se si può presupporre avesse un andamento abbastanza regolare, nonostante tutta la città si sia espansa in prossimità del pendio del quartiere Castello (Colavitti - Deplano 2002, 1120). Da questo quadro emerge che Cagliari era una città molto importante per il sud Sardegna, ricca, prospera, grazie soprattutto al suo porto e al suo entroterra fertile. Nonostante la sua importanza nell'antichità Cagliari è stata poco indagata archeologicamente, vista la sovrapposizione di più epoche e l'intensiva edilizia in tempi recenti. Per questo motivo ci restano che poche tracce della sua cultura musiva ${ }^{1}$.

\section{Le Terme in Localita' Bonaria}

Purtroppo non rimane più alcuna traccia di questo edificio scoperto nel 1907, però grazie alle accurate descrizioni di Antonio Taramelli (Taramelli, 1909, 135-147) sappiamo che il complesso era dotato di una grande sala con vasche e pozzetto centrale, con ad ovest diversi vani accessori. Queste

1 Verranno presi in esame 20 pavimenti, escludendo dunque quelli andati perduti, nonostante esistano foto d'archivio o frammenti troppo piccoli per essere interpretati. 
terme non erano collocate nel centro della città ma spostate ad oriente, questo potrebbe far pensare che non dovevano essere di grandi dimensioni.

- Pavimento 1

L'ambiente centrale era completamente mosaicato e misurava $8 \times 8$ metri. Il pavimento si trova oggi frammentato in diversi frammenti di cui:

a) 9 frammenti di 1) $0,94 \times 0,72$ metri; 2) 0,92 x 0,65 metri; 3) $0,83 \times 0,62$ metri; 4) $0,82 \times 0,63$ metri; 5) $0,82 \times 0,60$ metri; 6) $0,77 \times 0,68$ metri; 7) $0,74 \times 0,61$ metri; 8) 0,74 x 0,56 metri; 9) 0,70 $\mathrm{x} 0,54$ metri. Le tessere sono: bianche, ocra, grigie, nere e rosse di 0,8-1,2 x 1-1,4 cm. I frammenti sono stati staccati e sarciti di cemento nelle lacune, e si trovano presso il Museo Nazionale Archeologico di Cagliari senza numero di inventario, in discreto stato di conservazione.

b) Frammento di 0,97 × 0,77 metri, tessere bianche, nere, ocra, grigie e rosse di $0,5-1 \times 1 \mathrm{~cm}$. Numerose lacune, presso il Museo Nazionale Archeologico di Cagliari senza numero di inventario, in discreto stato di conservazione.

c) Frammento di $0,97 \times 0,70$ metri, tessere bianche, nere, ocra, grigie, rosse rosa di $0,6 \mathrm{x}$ 0,6-1 cm. Restaurato, si trova presso il Museo Nazionale Archeologico di Cagliari senza numero di inventario, in discreto stato di conservazione.

d) $\mathrm{Ne}$ resta solo una fotografia della Sopr. Archeologica di Cagliari e Oristano, neg. 676.

e) Frammento di $0,95 \times 0,87$, con tessere bianche, nere, ocra, rosse di 0,5-1 x $1 \mathrm{~cm}$. Restaurato, collocato presso il Museo Nazionale Archeologico di Cagliari senza numero di inventario, in buono stato di conservazione.

f) Frammento di $0,53 \times 0,53$ metri con tessere bianche, brune, nere, ocra di $0,6 \times 0,5-1 \mathrm{~cm}$. Abbondantemente sarcito di cemento, si trova presso il Museo Nazionale Archeologico di Cagliari senza numero di inventario, in cattivo stato di conservazione.

g) $\mathrm{Ne}$ resta solo una fotografia della Sopr. Archeologica di Cagliari e Oristano, neg. 645.

h) $\mathrm{Ne}$ resta solo una fotografia della Sopr. Archeologica di Cagliari e Oristano, neg. 643.

i) $\mathrm{Ne}$ resta solo una fotografia della Sopr. Archeologica di Cagliari e Oristano, neg. 678.

1) Frammento di $0,87 \times 0,72$ metri, con tessere bianche, ocra e marroni di $1,4 \times 1,5 \mathrm{~cm}$. Restauro in antico non ottimale, conservato presso il Museo
Nazionale Archeologico di Cagliari senza numero di inventario, in discreto stato di conservazione.

m) Frammento di 1,04 x 0,81 metri, con tessere bianche nere, rosse, grigie, ocra di 0,6 x 0,6-1 cm. Si trova presso il Museo Nazionale Archeologico di Cagliari senza numero di inventario, in buono stato di conservazione.

n) Frammento di $0,89 \times 0,68$ metri, con tessere bianche, nere, rosse, ocra e grigie di $0,6 \times 0,6-1 \mathrm{~cm}$. Sarcito in cemento nelle numerose lacune, collocato presso il Museo Nazionale Archeologico di Cagliari senza numero di inventario, in buono stato di conservazione.

o) Frammento di $0,84 \times 0,78$ metri, con tessere bianche, nere, gialle, ocra e rosse di $1 \mathrm{x}$ $1,2 \mathrm{~cm}$. Probabilmente restaurato in antico con tessere più piccole, custodito presso il Museo Nazionale Archeologico di Cagliari senza numero di inventario, in buono stato di conservazione.

p) Frammento di 0,67 x 0,55 metri, con tessere bianche, ocra, nere, rosse, grigie e rosa di 0,6 x $0,7-$ $1 \mathrm{~cm}$. Numerose lacune sarcite in cemento, si trova presso il Museo Nazionale Archeologico di Cagliari senza numero di inventario, in precario stato di conservazione.

q) Frammento di 0,94 x 082 metri, con tessere bianche, marroni, nere, grigie, verdi, ocra, rosse e rosa di $0,6 \times 0,7-1 \mathrm{~cm}$. Restaurato in antico con tessere più grandi e lastrine di marmo. Custodito presso il Museo Nazionale Archeologico di Cagliari senza numero di inventario, in cattivo stato di conservazione.

Il pavimento era decorato da uno schema a riquadri formati da fasce perpendicolari. Entro un bordo esterno a greca il campo era diviso da fasce decorate con treccia a otto elementi che andava a formare venticinque riquadri, quello centrale era occupato da un pozzetto circolare, nel quale si adeguavano i motivi della decorazione. Senza un criterio logico si alternavano, nei ventiquattro riquadri rimanenti, fiori e soggetti di tiaso marino. Subì un maldestro restauro in antico, nel quale vennero utilizzate lastre di marmo e tessere decisamente più grandi. Il frammento $a$ appartiene alle fasce che bordavano i riquadri, una treccia multipla bianca, nera, ocra, rossa e grigia (Fig. 1). Il frammento $b$ è un riquadro profilato di nero e da due linee dentellate bianche e nere, con fondo bianco nel quale si staglia un fiore a petali lanceolati, alle cui estremità si ha una foglia cuoriforme (Fig. 2). Il frammento $c$ presentava la bordatura a dentelli 


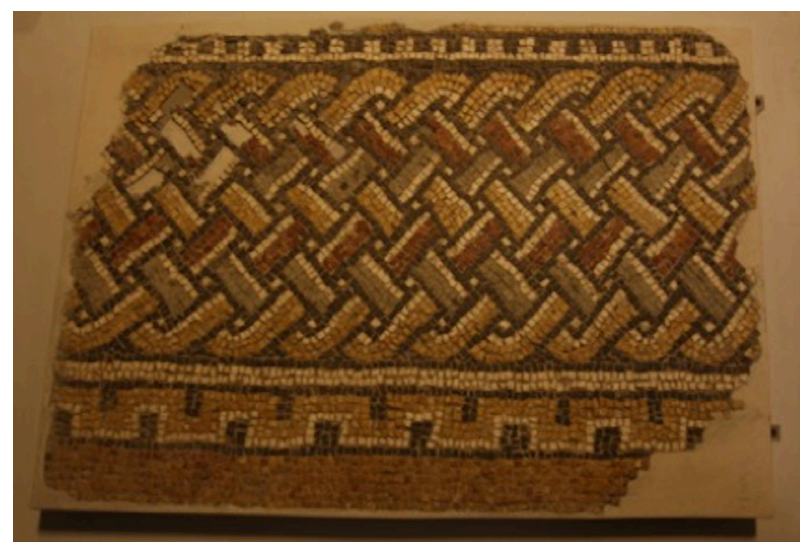

Figura 1 Pavimento 1, frammento $a$, dettaglio della cornice, Terme di Bonaria (Foto dell'Autore)

e nel campo si intravede il muso e parte del corpo di un delfino sul quale sta un erote, con gamba sinistra distesa e destra piegata, e con la mano sinistra regge le redini e con la destra una frusta, ha le ali spiegate, le linee del mare sono trattate in maniera semplicistica con tratti neri e grigi (Fig.3). La figura, come negli altri riquadri, è marcata da una pesante linea scura, che ne sottolinea i dettagli anatomici. Il frammento $d$ ha al suo interno la rappresentazione di una nereide che tiene un velo sopra il capo, seduta su un mostro dalla coda trifida (Fig. 4). Il frammento $e$ presenta la solita cornice a dentelli e al suo interno un fiore con quattro petali campaniformi e tricuspidati, agli angoli girali vegetali neri (Fig. 5). Nel frammento $f$ si scorge parte della cornice a treccia e parti anatomiche di un mostro marino con coda trifida. Il frammento $g$

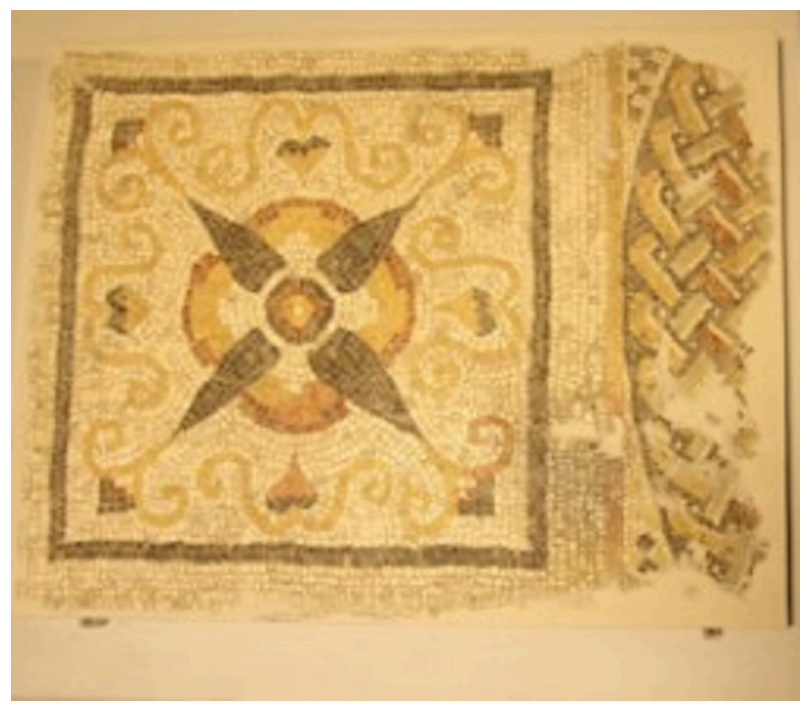

Figura 2 Pavimento 1, frammento $b$, Terme di Bonaria (Foto dell'Autore)

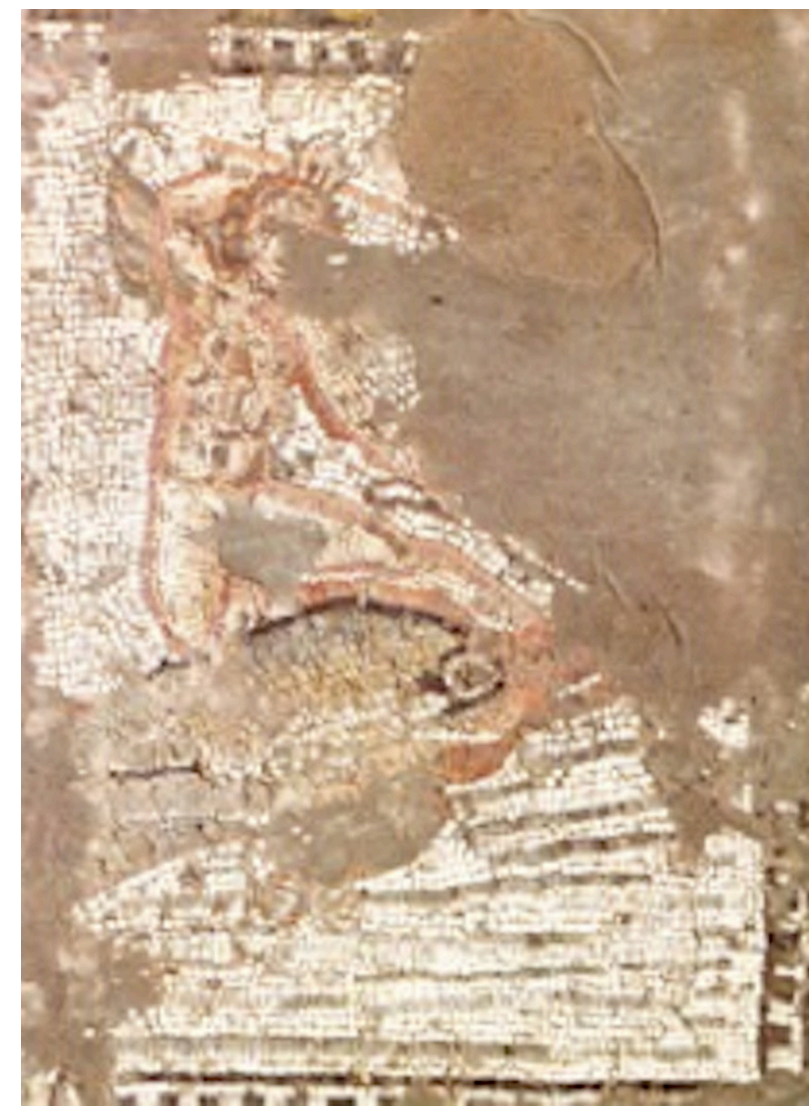

Figura 3 Pavimento 1, frammento $c$, erote su delfino, Terme di Bonaria (da Angiolillo 1981)

contiene la raffigurazione di un erote che guida un delfino con la coda bifida. L'erote ha le ali spiegate e ha una clamida che gli cinge la vita. Il frammento $h$ appartiene ad un riquadro con cornice a dentelli, e al suo interno un fiore formato da quattro petali che si aprono a loro volta in due foglie con bocciolo lanceolato. Agli angoli un bocciolo cuoriforme con due foglie a voluta. Il frammento $i$ è interessato dal solito bordo a dentelli e al centro del riquadro troviamo un tritone.

Il mostroè barbatoe possiedefolti capelli, i muscoli sono resi anatomicamente con bruschi passaggi di colore, tiene nella mano sinistra un'ancora e con la destra regge la marra della stessa. Nel frammento $l$ si scorge un motivo floreale irriconoscibile dal maldestro restauro in antico. Il frammento $m$ si trovava adiacente al pozzetto centrale e dunque ha una treccia che assume un andamento circolare, al suo interno ha una composizione vegetale abbastanza dettagliata: una corona circolare con centro nero, rosso e ocra, dal quale si staccano petali lanceolati in numero di quattro, che terminano con un bocciolo rosso e nero. Negli spazi tra i 
petali yi sono foglie cuoriformi con due racemi a volute ocra. Il frammento $n$ possiede al suo interno parte della treccia a più elementi, il campo risulta quasi del tutto illeggibile, ma pare che si possa scorgere un erote. Il frammento $o$ presenta la stessa decorazione del frammento $m$. Nel frammento $p$ si intravede un erote con la gamba sinistra piegata sul dorso di un mostro marino, presumibilmente un delfino. La nereide del frammento $q$ è svestita, il braccio destro piegato e con la mano tiene un velo che le gira intorno alle spalle, sul dorso di un mostro marino del quale si riesce a scorgere solo la coda trifida. Per quanto riguarda gli altri riquadri A. Taramelli fornisce la descrizione, e dunque sappiamo che erano presenti un cervo con erote, un erote con ali spiegate sul dorso di un delfino, una rosetta, un serpente marino con sopra un erote, un erote sul dorso di un delfino con la coda trifida, un erote sopra un mostro marino, una rosa, un mostro marino con le corna a chele di granchio e del marmo bianco con delle rosette. La disposizione dei riquadri non segue un filo logico, poiché accanto a figure tipiche del tiaso marino abbiamo figure vegetali quali rosette. Lo schema a riquadri è ampiamente utilizzato dai musivari romani in tutto l'Impero con scene e soggetti differenti, a Teramo col famoso Mosaico del Leone datato al I secolo a.C. (Angeletti, 1997, 45), nel pavimento della Villa del Mito, a Sant'Angelo di Vado, non datato ma appartenente alla struttura del I secolo d.C. (De Marinis, 2009, 12) o per esempio in un pavimento di Italica troviamo trentatré riquadri raffiguranti uccelli virgula datato al II secolo d.C. (Quattrocchi, 2011, 51-53), nella famosa Villa del Casale, nella Sala del Mosaico a riquadri in un pavimento datato al

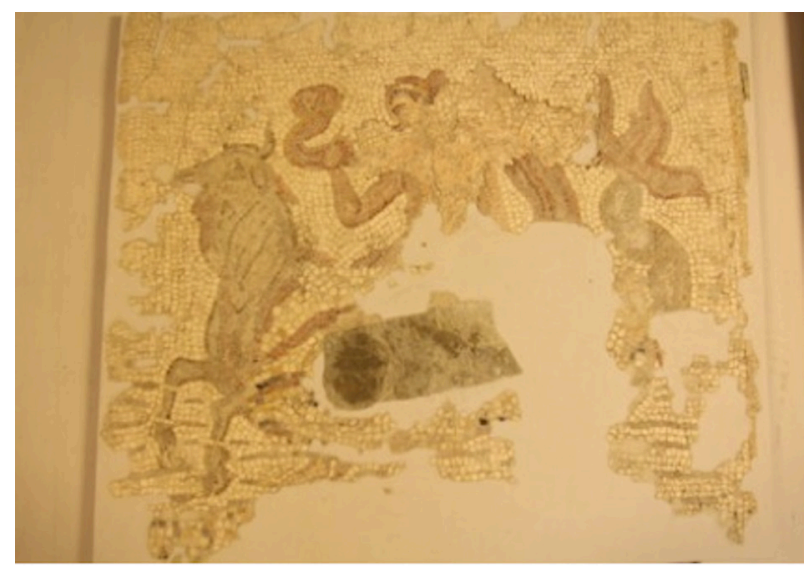

Figura 4 Pavimento 1 , frammento $d$, nereide, Terme di Bonaria (Foto dell'Autore)
IV secolo d.C. (Pace, 1955, 56). In ambiente africano sono da segnalare gli esemplari di Bulla Regia, con datazioni che oscillano dalla fine del II secolo sino a pieno IV secolo (Hanoune, 1969, 261; 296; 329), e i pavimenti di Volubilis, soprattutto quello delle Terme, datato al III secolo d.C. (Zehnacker-Hallier, 1964, 404). In queste composizioni abbiamo l'esatta commistione tra la scuola occidentale, dedita al geometrismo, e la scuola orientale, che concentrava la sua attenzione su temi figurati. Nel caso di questo pavimento calaritano ci troviamo di fronte ad un thiasos marino smembrato in più quadretti, con i personaggi principali del tema, come l'erote o la nereide. L'iconografia dell'erote ha un vastissimo repertorio, lo ritroviamo in tantissimi pavimenti, sia nella sua connotazione marina, dunque sopra un delfino, sia terrestre. In territorio iberico, a Elce, nel mosaico di Bacco bambino si hanno eroti intorno al dio, il pavimento è datato alla fine del I secolo d.C. (López Monteagudo, 2008, 2557). In un triclinio di Volubilis troviamo eroti in un pavimento del II-III secolo (Blázquez Martínez, 2006, 1396), a Lixus nel famoso mosaico di Venere e Adone, della fine II inizi III secolo (Blázquez Martínez, 2006, 1409), e ad Utica, nella Casa di Catone del III secolo (Neira Jiménez, 2006, 1540), ed un esemplare più tardo, a Luni del IV secolo (López Monteagudo, 2008, 2587). Ovviamente con la presa di potere dei cristiani l'iconografia dell'erote tramuterà, e verrà riutilizzato in veste cristiana $e$ non più pagana. Anche l'iconografia della donna nuda o semi-nuda, ammantata, o vestita, seduta sul dorso di un animale marino, il più delle volte a cavalcioni su di esso, che generalmente definiamo

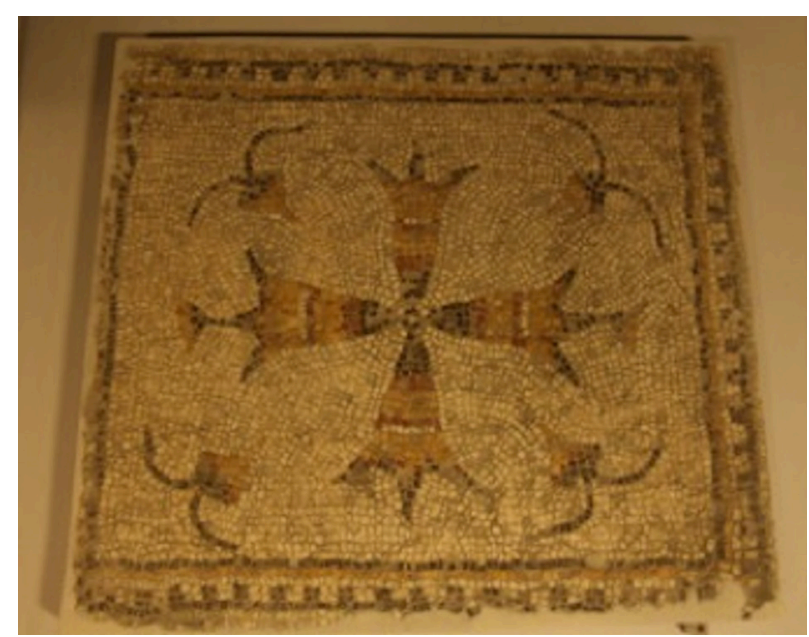

Figura 5 Pavimento 1, frammento e, Terme di Bonaria (Foto dell'Autore) 
Nereidi è ben attestata. Fanno la loro comparsa in ambiente ellenistico-romano, su tessellati del IV a.C. (Dumbabin, 1978, 154-158) e presenti sino almeno al IV secolo d.C. (Rinaldi, 2011a, 222) e vedono una maggiore fioritura, soprattutto a Ostia, nel II secolo d.C. (Teatini, 2007, 647-648), ma la figura delle Nereide viene utilizzata anche in altri contesti, come ad esempio in una placchetta in osso di Castellu, in Corsica, che ha strettissime somiglianze con una placchetta, sempre in osso, ritrovata a Thugga, e datata al V secolo d.C. (Vismara, 1984, 182). La resa dei volti è grossolana, e l'anatomia resa in maniera semplicistica e frettolosa, lo stesso discorso vale per gli animali marini, che sembravo appena abbozzati. La tipologia dei fiori ci riporta ad esemplari africani del III secolo d.C. (Parlasca, 1959, 19). La grossolanità della trattazione, e la scelta di elementi di contorno, riempitivi, cornici, che non rispettano nessuna coerenza compositiva, obbliga a datare questo pavimento alla metà del III secolo d.C, probabilmente realizzato da una bottega locale.

\section{LA FULLONICA}

Durante i lavori per l'ampliamento della sede dell' INPS, tra via XX Settembre e viale Regina Margherita, si trovarono resti di un impianto artigianale-produttivo di età repubblicana. Non fu possibile approfondire l'indagine archeologica, che risente della mancanza di dati stratigrafici. Si trattava di un edificio al cui interno vi era un pozzo, una vasca fiancheggiata da due anfore, e una serie di vasche di diverse dimensioni, che fece pensare che si trattasse di una "fullonica", ovvero un impianto per la trattazione dei tessuti, in base ad alcuni confronti di edifici simili come ad Ostia (Becatti, 1961, 134). Non si sa quanto tempo restò in funzione, ma ci fu almeno una fase di abbandono, testimoniata da un accumulo di terra, sopra il quale si installarono edifici di età tardo-romana e successivamente bizantina, in più adiacente all'edificio vi era una struttura punica. In un rilievo dell'epoca degli scavi si vede una torretta quadrangolare, formata da blocchi litici di riutilizzo, che potrebbe far pensare ad una struttura difensiva fortificata.

\section{- Pavimento 2}

Il pavimento (Fig. 6) intorno alla vasca misura $1,60 \times 0,89$ metri nel quale troviamo un'iscrizione con lettere alte $14,5 \mathrm{~cm}$. Le tessere utilizzate sono bianche, rosse, nere e verdi di $0,8 \times 1 \mathrm{~cm}$. Si trova in situ, ma solo la parte dell'iscrizione è visibile, in discreto stato di conservazione. La fascia che corre

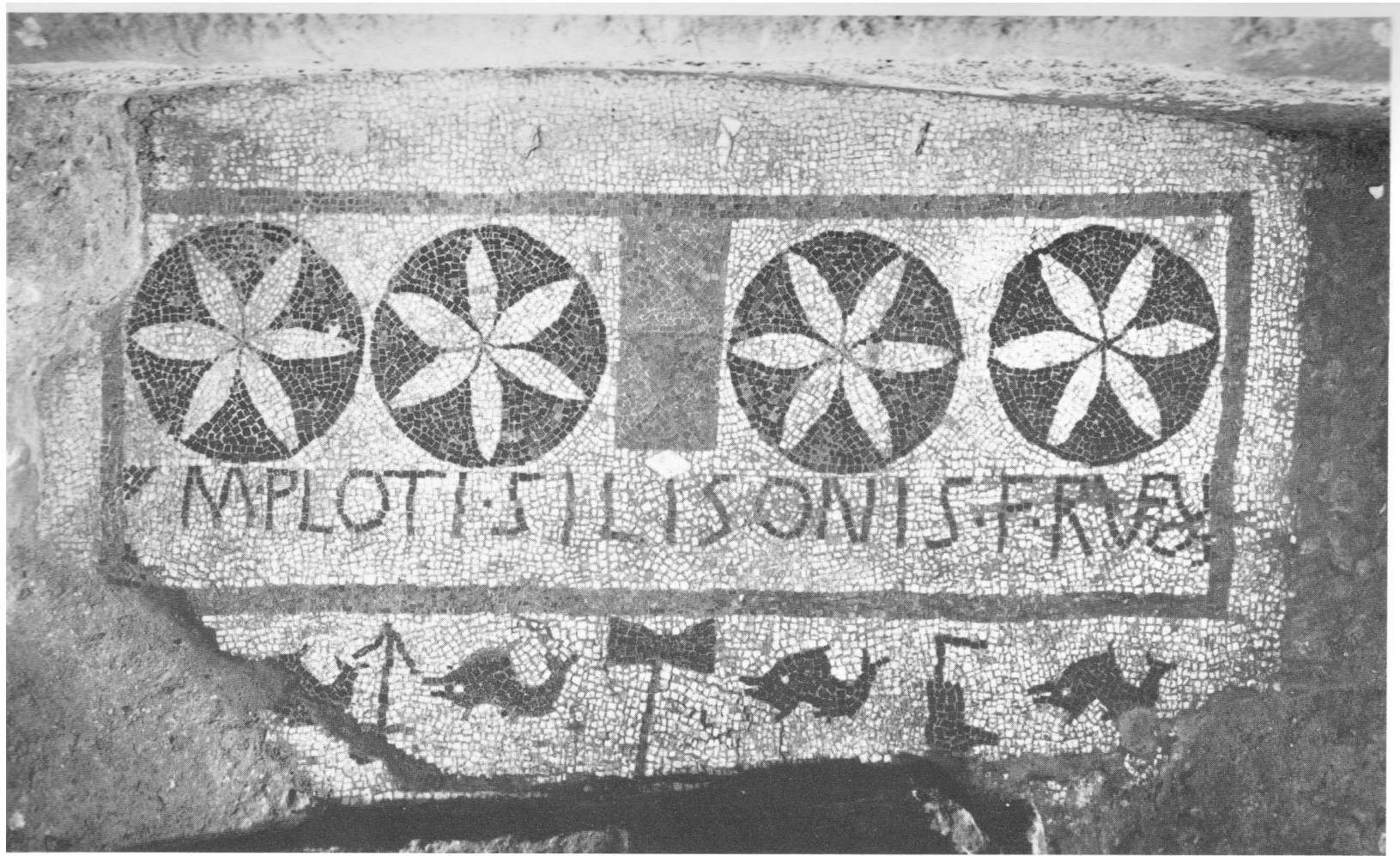

Figura 6 Pavimento 2, iscrizione, Fullonica (da Angiolillo 1981) 
lungo la vasca presenta un fondo bianco, nel quale si alternano piccoli delfini in tessere nere, separati da un'ancora, da una bipenne, da un timone. Su di un lato della vasca il mosaico si amplia e presenta un campo decorato da due gruppi di cerchi separati da una fascia. I cerchi hanno un campo nero e al loro interno si trova inscritto un fiore di sei foglie lanceolate. Al di sotto di questi due gruppi troviamo l'iscrizione, in tessere nere:

M(arcus) Ploti(us) Silisonis f(ilius) Rufus

Dunque Marco Plotio Rufo figlio di Silisone. Sopra la seconda $i$ di Silisonis troviamo una lastrina romboidale in marmo (Quattrocchi, 2015).

Gli elementi che compaiono in questo pavimento sono prevalentemente utilizzati durante l'età repubblicana e la prima età imperiale. Il fiore a sei petali lanceolati lo troviamo attestato ad Ostia, nella Domus a peristilio, agli inizi del I secolo d.C. (Becatti, 1961, 69), nella Villa Adriana alla metà del I secolo a.C. (Lugli, 1927, 194), a Délos ritroviamo sia il fiore a sei petali lanceolati che le bipenni e i delfini, con datazione tra il II ed il I a.C. (Bruneau, 1972, 102-104), e sempre in Italia, nella città romana di Claterna, dove i fiori sono in gruppo di quattro, identici a quelli della nostra soglia, e sono datati alla fine del I secolo a.C. (Conti, 2012, 2-3). Oltretutto da notare che l'iscrizione ci fornisce un altro elemento datante: infatti è presente il nominativo in $-i$ del nomen, una caratteristica utilizzata sino al 100 a.C. e che svanisce in età giulio-claudia (Kaimio, 1969, 27). Un altro dettaglio emerge dall'iscrizione: il cognomen Silisonis, è stato identificato da F. Vattioni (Vattioni, 1979, 185) come punico, e R. Zucca (Zucca, 1996, 1459-1460) lo rimanda al cognomen Siliso, derivante da un sls punico con significato di tre, in pratica un Tertius latino. Mi trovo in disaccordo con questa tesi, poiché questo cognomen lo ritroviamo prevalentemente in Italia centrale, nella zona osco-umbra, regione che non ha traccia di insediamenti punici; credo invece che si potrebbe ricollegare sempre all'ambiente cartaginese, ma derivante dall'aggettivo della I classe sīlus-aum, con significato "dal naso camuso", dunque un soprannome dato per connotare qualcuno di discendenza punica, un sardo-punico in definitiva. Questo non dovrebbe stupire, perché ricordiamo che Cagliari ha un substrato punico molto forte, dunque è pensabile che al momento della creazione della provincia Sardinia et Corsica, e successivamente con l'elevazione al rango di municipio da parte di Cesare alla città di Caralis avvenuta dopo la guerra civile, gli abitanti di origine punica o sardo-punica abbiano ottenuto un cognomen per eguagliarsi ai cittadini romani. Il pavimento si data tra la fine dell'età repubblicana e gli inizi dell'Impero.

\section{La Villa di Tigellio}

Il complesso residenziale noto come la Villa di Tigellio è in realtà un agglomerato di tre domus: la Casa degli Stucchi, la Casa del Tablino dipinto e una terza abitazione. Le abitazioni sorgono in una zona con impianto ortogonale e si inseriscono nella rete viaria perfettamente. L'ingresso era su una via, non messa in luce, che corre parallela al corso V. Emanuele. La Casa degli Stucchi è parzialmente scavata, sul fianco destro del suo atrio, che era dotato di impluvium, si aprono una serie di ambientini, probabilmente dei cubicula, mentre sul fondo, di fronte all'ingresso principale, vi è un grosso tablino. Il tablino è affiancato da due stanze, una a destra e una a sinistra, dove vi sono tracce di scale per il piano superiore. Il tablino, in una seconda fase edilizia, venne ridotto, poiché ritroviamo un muro che utilizza la tecnica degli ambienti aggiunti più tardi, una tecnica "a telaio" (Angiolillo, 1987, 92). $\mathrm{Fu}$ compiuto un saggio stratigrafico, alla ricerca di materiale datante, ma diete esito negativo. Nella stessa fase costruttiva troviamo una nuova pavimentazione e una nuova decorazione parietale. La Casa degli Stucchi è affiancata a nord-ovest da un'abitazione autonoma, almeno in una seconda fase, perché gli scavi hanno evidenziato un corridoio che metteva in comunicazione le due abitazioni, dunque è presumibile che al momento della costruzione fosse un'unica domus, divisa in due abitazioni separate in epoca più tarda. Nettamente separata risulta invece essere la Casa del Tablino dipinto, che presenta un ingresso decentrato. I saggi di scavo hanno evidenziato che il tablino fosse chiuso verso l'atrio, fin dalla sua prima edificazione. La planimetria della prima fase edilizia non si conosce, anche se possiamo datarla all'ultimo quarto del I secolo d.C., mentre per la seconda fase, attribuibile al I secolo d.C., si hanno più notizie: venne aumentato il volume generale dell'abitazione con l'aggiunta di vani secondari nella parte nord-est, e la corte interna venne ingrandita in seguito all'abbattimento di pareti e venne eretto il compluvium, retto da quattro colonne, inoltre venne edificato un piano superiore, del quale non si ha traccia se non per dei gradini che si affacciavano direttamente sulla corte. 


\section{- Pavimento 3}

Di questo pavimento (Fig. 7) resta solo un emblema delle dimensioni di 0,55 x 0,54 metri, in tessere bianche, rosse, nere, ocra, azzurre, verdi, grigie, marroni di 0,1-0,4 cm di lato. Una lacuna è sarcita di cemento. Conservato presso il Museo Archeologico Nazionale di Cagliari, in uno stato di conservazione precario.

Il bordo è formato da una cornice dentellata bianca e nera, il campo è grigio chiaro con una sottile fascia rosa che indica il suolo. All'interno del campo troviamo un cesto di vimini, marrone e ocra, colmo di frutti rossi. A sinistra del cesto troviamo un gallinaceo, con lunghe zampe rosse e l'occhio azzurro, dal piumaggio marrone, nell'atto di beccare i frutti, al di sopra di questo volatile se ne scorge un altro, non identificabile perché è presente una grossa lacuna.

Questo emblema trova un confronto diretto con il pavimento contenente il Mosaico di Orfeo a Leptis Magna, della fine del II secolo d.C. (Guidi, 1935, 30), nel quale troviamo la stessa ripartizione dello spazio interno, dunque quattro zone decorate a chiasmo, con il soggetto però reso in maniera speculare. L'iconografia è praticamente identica, e la ritroviamo anche a Zliten in un mosaico delle Stagioni, dello stesso periodo (Aurigemma, 1960, 115); l'iconografia identica potrebbe far pensare che si tratti di uno stesso cartone replicato, ovvero

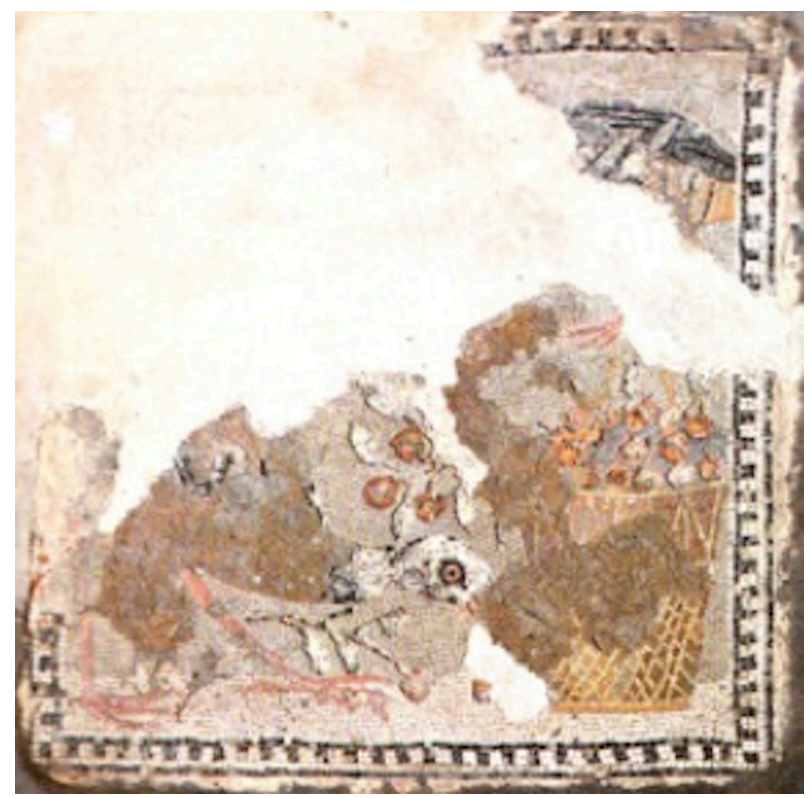

Figura 7 Pavimento 3, gallinaceo e cesto con frutti, Villa di Tigellio (da Angiolillo 1981) di un copy book di riferimento che i musivari utilizzavano per realizzare questi pavimenti. Questo emblema va datato al III secolo d.C., ed è eseguito da chiare maestranze africane.

\section{- Pavimento 4}

Anche in questo caso abbiamo solo l' emblema di 0,55 x 0,55 metri. Tessere bianche, rosa, nere, grigie, marroni, verdi, gialle, azzurre, ocra, di 0,1$0,4 \mathrm{~cm}$ di lato. Una lacuna, sarcita di cemento, si ha nella parte superiore. Conservato presso il Museo Archeologico Nazionale di Cagliari, in precario stato di conservazione. Il bordo presenta una cornice a dentelli bianchi e neri, e il campo è decorato da un'anatra col becco aperto, protesa a beccare o bere dal cratere centrale. Una seconda anatra ha il capo piegato all'indietro (Fig. 8). I volatili hanno il becco bianco e rosa, e il piumaggio è variopinto con diverse striature gialle e nere, e sono profilate da una linea di contorno marrone. Si intravedono altri due animali, ma non si riesce a capire quali siano, avendo gli stessi colori delle due anatre si potrebbe pensare che in totale vi siano quattro volatili identificabili come anatre. Al centro del campo vi è un cratere, ocra, con ombreggiature marroni. Il fondo è grigio, rosato in prossimità del suolo. Anche questo pavimento si ricollega a quello precedente. In questo caso però abbiamo una reinterpretazione del famoso mosaico delle

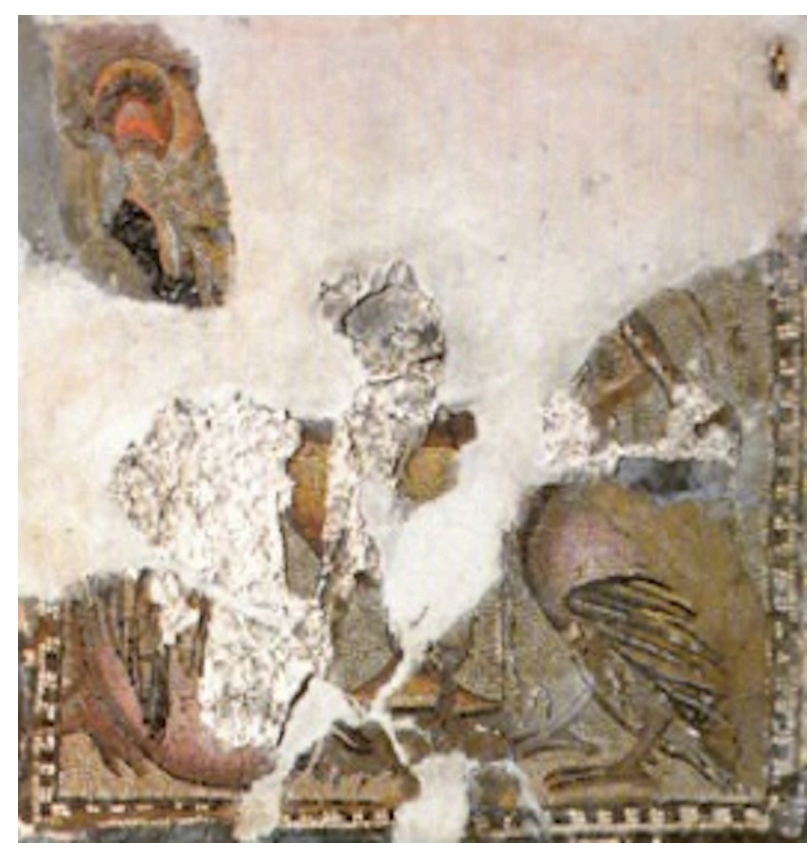

Figura 8 Pavimento 4, anatra, Villa di Tigellio (da Angiolillo 1981) 
colombe di Sosos. Uno schema simile lo ritroviamo a Leptis Magna nella Villa fuori Porta Lebda, della fine del II secolo d.C. (Aurigemma, 1960, 50). Anche questo pavimento va datato al III secolo d.C.

\section{- Pavimento 5}

Resta solo un frammento di quello che in antico era un emblema, con dimensioni di 0,31 x 0,19 metri, con tessere bianche, nere, ocra, grigie, rosa, rosse, gialle e verdi delle dimensioni di $0,1-0,4 \mathrm{~cm}$ di lato. Lacunoso nell'angolo inferiore destro. Custodito nei magazzini del Museo Archeologico Nazionale di Cagliari, in precario stato di conservazione. Il campo è bordato da una doppia linea, nera e bianca, e presenta un pesce con diverse sfumature. Il pesce è legato per la coda e ha la bocca aperta, il muso, probabilmente per un errore del musivario, tocca il bordo superiore del riquadro (Fig.9).

\section{- Pavimento 6}

Anche in questo caso ci troviamo di fronte ad un emblema di 0,55 x 0,56 metri. Tessere bianche, nere, marroni, rosa, brune, ocra, grigie e blu, di 0,1$0,4 \mathrm{~cm}$ di lato. Ampia lacuna che colpisce la parte superiore, conservato presso il Museo Archeologico Nazionale di Cagliari, estremamente precario stato di conservazione. All'interno di un doppio bordo, marrone e bianco vi è il campo, decorato da un pesce in posizione arcuata, con la coda tenuta da un legaccio, con ai lati due ricci di mare neri e blu (Fig. 10). Molto accurati sono i dettagli, l'anatomia del pesce è resa in maniera magistrale dal diverso uso di tessere di varia colorazione, e con sapienti espedienti per creare porzioni di luce ed ombre sul suolo.

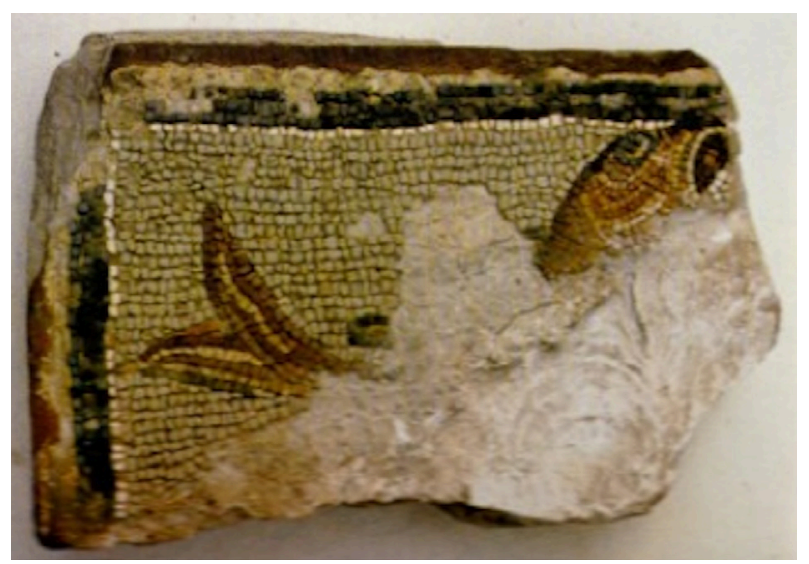

Figura 9 Pavimento 5, pesce, Villa di Tigellio (da Angiolillo 1981)

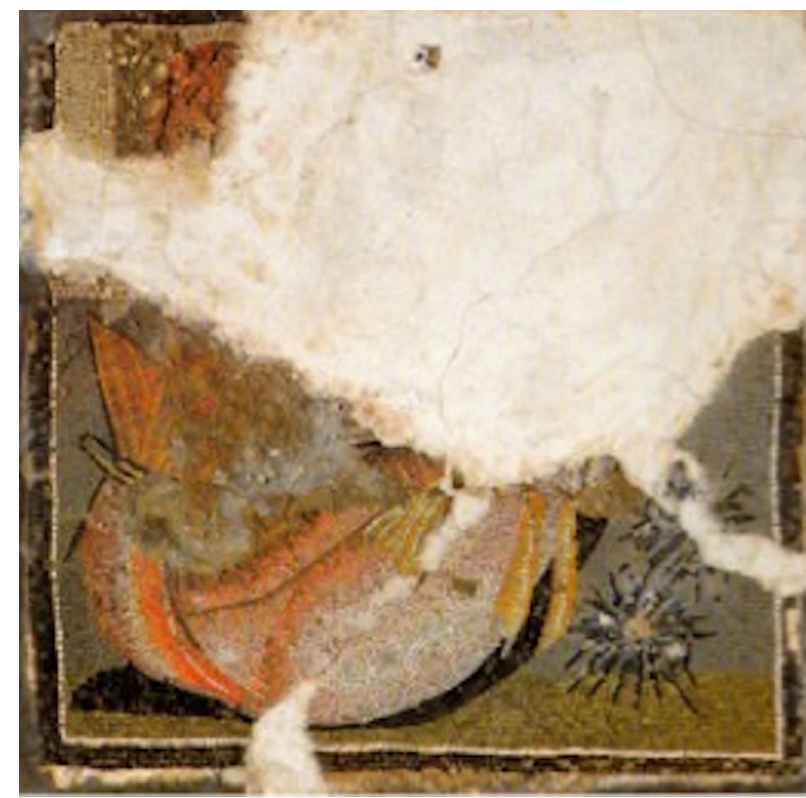

Figura 10, Pavimento 6, pesce e ricci, Villa di Tigellio (da Angiolillo 1981)

A Sousse troviamo un notevole confronto iconografico del pesce, in un pavimento datato alla fine del II secolo d.C. (Foucher, 1961, 115), ma i confronti maggiori li abbiamo non in pavimenti musivi, ma in alcune pitture di I stile a Pompei (Pernice, 1938, 149-152), infatti vi è la stessa resa anatomica e la stessa attenzione per la luminosità del riquadro, in più in entrambi i casi abbiamo la stessa fusione del colore resa grazie ad una sottile dentellatura.

- Pavimento 7

L' emblema misura $0,55 \times 0,55$ metri, con tessere bianche, rosse, rosa, nere e brune di 0,1-0,4 cm di lato. Risulta danneggiato da una lacuna che occupa la maggior parte del riquadro. Conservato presso il Museo Archeologico Nazionale di Cagliari, in precario stato di conservazione. Il bordo è formato da una cornice a dentelli bianchi e neri, e profila un campo grigio, dove, per via della grossa lacuna, sono leggibili solo delle zampe rosse, probabilmente di un gallinaceo (Fig. 11). Doveva trattarsi di una decorazione simile ai pavimenti nn. 3-4.

- Pavimento 8

Emblema di 0,54 x 0,54 metri, con tessere bianche, beige, nere, rosa, azzurre, rosse e grigie, di $0,1-0,4 \mathrm{~cm}$ di lato. Presenta una grossa lacuna centrale. Conservato presso il Museo Archeologico Nazionale 


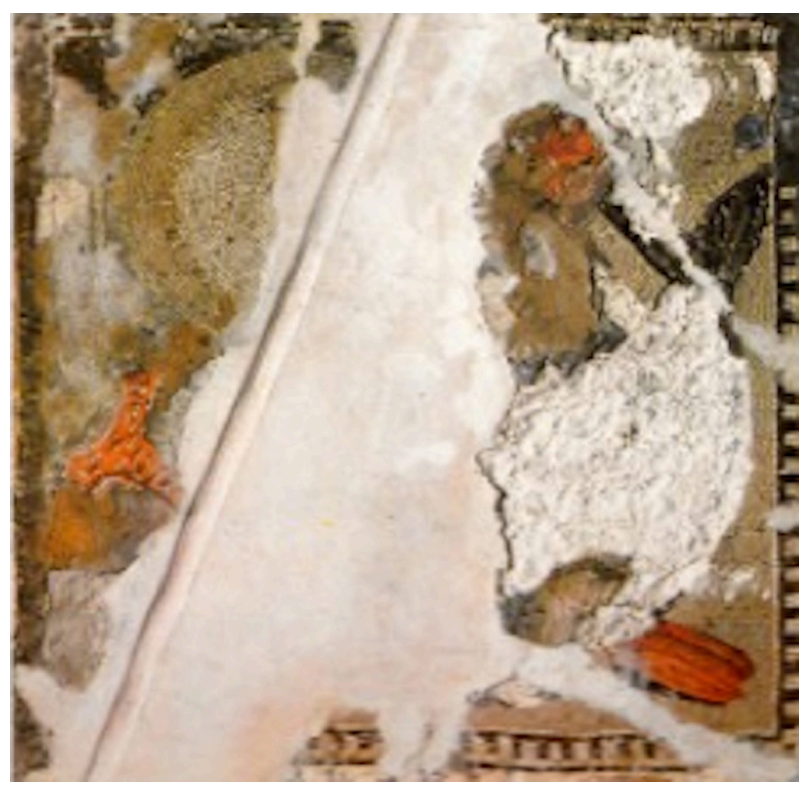

Figura 11, Pavimento 7, gallinacei, Villa di Tigellio (da Angiolillo 1981)

di Cagliari, in pessimo stato di conservazione. Una cornice a dentelli bianchi e neri profila un campo grigio, col suolo rosa, si intuisce la presenza di un pesce grazie alla pinna caudale e alla dorsale. Il resto del campo è illeggibile a causa della grossa lacuna. Non è possibile riportare confronti con questo pavimento per via della sua scarsa leggibilità.

\section{- Pavimento 9}

Questo pavimento si trovava nell'ambiente 16 della Casa degli Stucchi, e si tratta di un frammento di $0,51 \times 0,40$ metri, formato da tessere bianche e nere di $0,8-1,3 \times 1-1,3 \mathrm{~cm}$. Al centro della decorazione troviamo un disco in laterizio. In situ, in buono stato di conservazione. Un riquadro bianco (Fig. 12) è decorato da un cerchio in tessere nere, al suo interno un fiore a sei petali lanceolati bianchi con il centro rosso (disco in laterizio). Lo stesso tipo di fiore l'abbiamo ritrovato nella fullonica, nel pavimento n. 1 al quale si rimanda per i confronti. La datazione però è uguale, fine dell'età repubblicana - inizi dell'Impero.

\section{- Pavimento 10}

L'ambiente 19 era decorato da un pavimento di cui rimane un frammento di 1,87 x 0,59 metri, con l'utilizzo di tessere di colorazione bianca, ocra, nera, e grigia di 0,9-1,5 x 1-2 cm. In situ, in precario stato di conservazione. La banda di raccordo ocra viene seguita da un motivo a riquadri

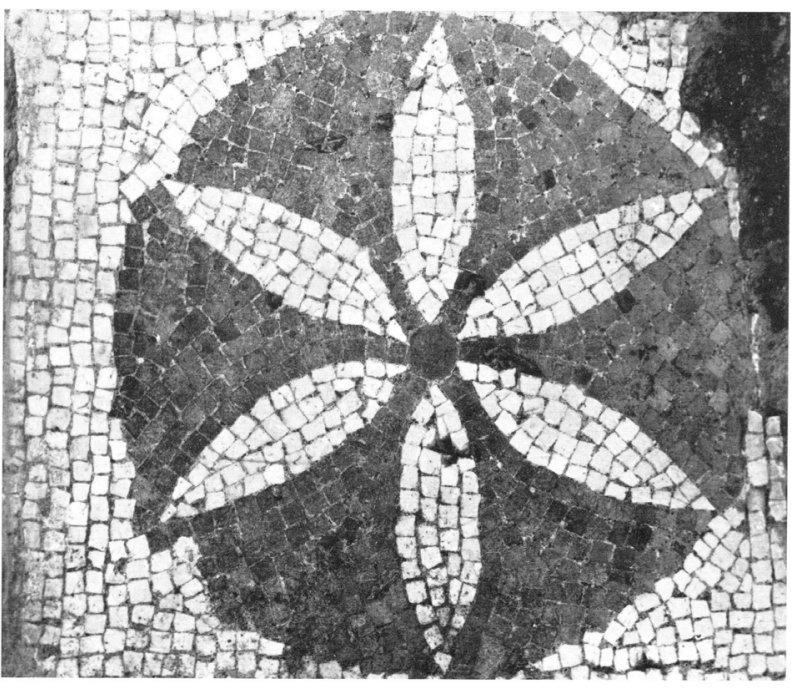

Fig. 12 Pavimento 9, fiorone a sei petali, Villa di Tigellio (da Angiolillo 1981)

che si formano dall'incrociarsi di fasce bianche con profilo nero. Lo schema segue una sola fila. Il punto di intersezione delle fasce è ocra, e dello stesso colore sono i quadrati di risulta dove, nell'unico integro, è presente un quadrato a lati concavi con al suo interno una croce di Malta. I confronti più vicini li abbiamo in due mosaici di Bulla Regia, il primo datato alla fine del II secolo d.C. (Hanoune, 1969, 128), il secondo lo troviamo nella Casa della Caccia ed è datato alla prima metà del III secolo d.C. (Hanoune, 1969, 248). Il pavimento fu messo in posa, con molta probabilità, tra la fine del II e gli inizi del III secolo d.C.

\section{- Pavimento 11}

Nell'ambiente 18 troviamo un frammento di pavimento di $0,51 \times 0,25$ metri, il vano in totale misurava 1,70 × 0,85 metri. Le tessere utilizzate sono bianche, grigie, nere, verdi, rosse e azzurre, di dimensioni pari a $0,5-1 \times 1-1,5 \mathrm{~cm}$. In situ, in precario stato di conservazione. La banda di raccordo è formata da una serie di fasce con tessere rosse, azzurre e bianche, adiacente alla banda vi è un bordo decorato da girali floreali neri su fondo bianco, che ricordano dei gigli. Il motivo presenta i fiori disposti alternativamente al dritto e al rovescio con foglie rosse e blu. Del campo non si è conservato niente. Un motivo con una delimitazione cronologica molto netta, infatti compare in Africa nel II secolo e scompare nel III secolo. Lo ritroviamo in un ambiente domestico, nella Casa di Nettuno ad Acholla, nell'ultimo 
quarto del II secolo d.C., e a Thysdrus nella Casa del Pavone, datato alla fine del II secolo (Foucher, 1963, 7), nel Mosaico del Nuotatore della prima metà del II secolo d.C. (Foucher, 1963, 79). Questo pavimento sarà dunque da datare non al più tardi del III secolo d.C.

\section{- Pavimento 12}

La soglia tra i vani 24 e 17 era decorata da un pavimento di $0,40 \times 0,05$ metri, in tessere bianche e nere di $1 \mathrm{~cm}$ di lato. In situ, in condizioni di conservazione estremamente precarie. Il pavimento si presenta come un semplice motivo a scacchiera, in cui ogni elemento della scacchiera stessa è rappresentato da una tessera. Questo schema è diffuso in tutta l'età repubblicana, con persistenze sino al II secolo d.C. Probabilmente questo fu tra i primi pavimenti a esser messo in posa e mai sostituito, come avvenne con gli altri, dunque sarà da datare non oltre al I secolo d.C.

- Pavimenti 3-8: osservazioni

In antico si trattava, evidentemente, di un unico pavimento, con una decorazione che definiamo a xenia, ovvero "doni ospitali", una tipologia di pavimento estremamente rara in tutta la Sardegna, eccezion fatta per il pavimento di Sordo (SS) in località Santa Filitica, dove è presente uno schema simile, ma non con temi figurativi animali ma bensì con oggetti per la toeletta (Angiolillo, 1986, 607-608). La datazione d'insieme dei pavimenti si attesta agli inizi del III secolo, in virtù anche dei confronti con il mosaico di Tripoli, degli inizi del II secolo d.C. (Romanelli, 1916, 344), e del Mosaico dei Gladiatori di Zliten, datato alla seconda metà del III secolo d.C. (Carandini, 1967, 114-116). Chiaramente ci troviamo di fronte a maestranze africane estremamente abili, perché le figure non risentono di alcun tipo di appesantimento, le anatomie sono rese con grande maestria, e vi è attenzione ai giochi di luce e ombre dei riquadri. Unitariamente possiamo datare questi pavimenti nel primo quarto del III secolo d.C.

\section{La CASA DEgli Emblemi Punici}

Questo edificio fu scavato da S. M. Puglisi nel 1943. I resti comprendono un atrio e tre ambienti che vengono identificati come tablinum e alae (Puglisi, 1943, 162). L'atrio ha una forma rettangolare di $3,90 \times 8,60$ metri, e in senso longitudinale, due colonne di calcare. Il cortiletto dunque non aveva una copertura sui quattro lati, tipico dell'architettura romana, ma soltanto lungo i lati brevi come spioventi. Il pavimento è leggermente inclinato, proprio per permettere alle acque piovane di defluire. Su un lato lungo dell'atrio si aprono i tre ambienti sopra citati. Il tablino ha una forma rettangolare di 3,20 x 4,65 metri, la muratura era in grossi blocchi squadrati, con minime tracce di intonaco. Delle alae rimangono poche tracce, tra cui alcuni muri perimetrale in blocchi squadrati. Generalmente viene identificata come un'abitazione romana (Puglisi, 1943, 162-165; Angiolillo, 1981, 105) ma devo dissentire da questa affermazione. Infatti ci troviamo di fronte ad una tipica casa punica, incentrata su una corte, a cui poi evidentemente sono stati annessi ambienti dal gusto romano. Infatti il cortile risulta essere nelle case puniche il nucleo della struttura domestica (Mezzolani, 2000, 1225) dal quale si diramano i diversi ambienti di rappresentanza o di servizio. E normale che poi l'abitazione venne abitata, successivamente, da cittadini romani, che ne modificarono la planimetria.

\section{- Pavimento 13}

Del tablino resta solo un frammento di pavimento di 0,74 metri di lato, in signino con tessere bianche di 1-1,3 x 1,3-1,4 cm. Conservato nel giardino del Museo Archeologico Nazionale di Cagliari, in buono stato di conservazione. In cementizio a base fittile con tessere in ordito regolare, ed alcune disposte in obliquo. Su un lato si nota la traccia, che bordava il tablino su un solo lato, di tessere bianche. Una datazione che si colloca nell'età repubblicana sembra corretta.

\section{- Pavimento 14}

Della pavimentazione dell'atrio si conservano due pannelli: $a$ ) di $0,78 \times 0,75 ; b)$ di $0,66 \times 0,52$, con tessere bianche di $0,8-1 \mathrm{~cm}$ di lato. Presso il giardino del Museo Archeologico Nazione di Cagliari, in buono stato di conservazione. Nei frammenti superstiti di questo cementizio a base fittile si sono conservate due figure: la prima è rappresentata dalla figura della dea Tanit, di figura triangolare con appendici rettilinee piegate ad angolo retto a raffigurare le braccia, mentre il capo è un cerchio appoggiato al vertice del quadrato (Fig. 13); la seconda figura è circolare, con due appendici piegate alle estremità ad angolo retto. La figura di Tanit la si trova in tutto il 


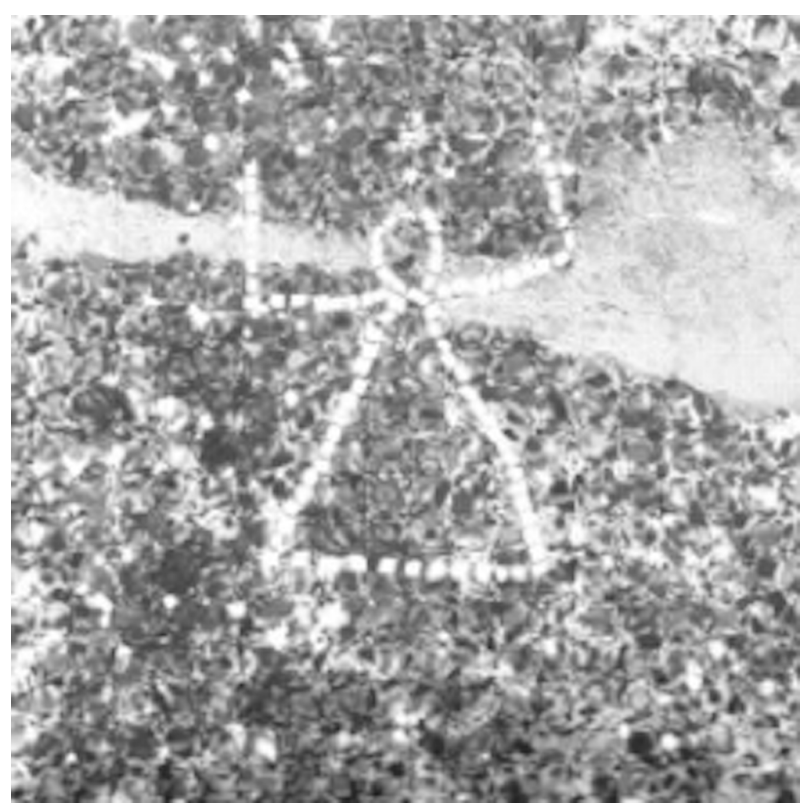

Fig. 13 Pavimento 14, dea Tanit, Casa degli emblemi punici (da Angiolillo 1981)
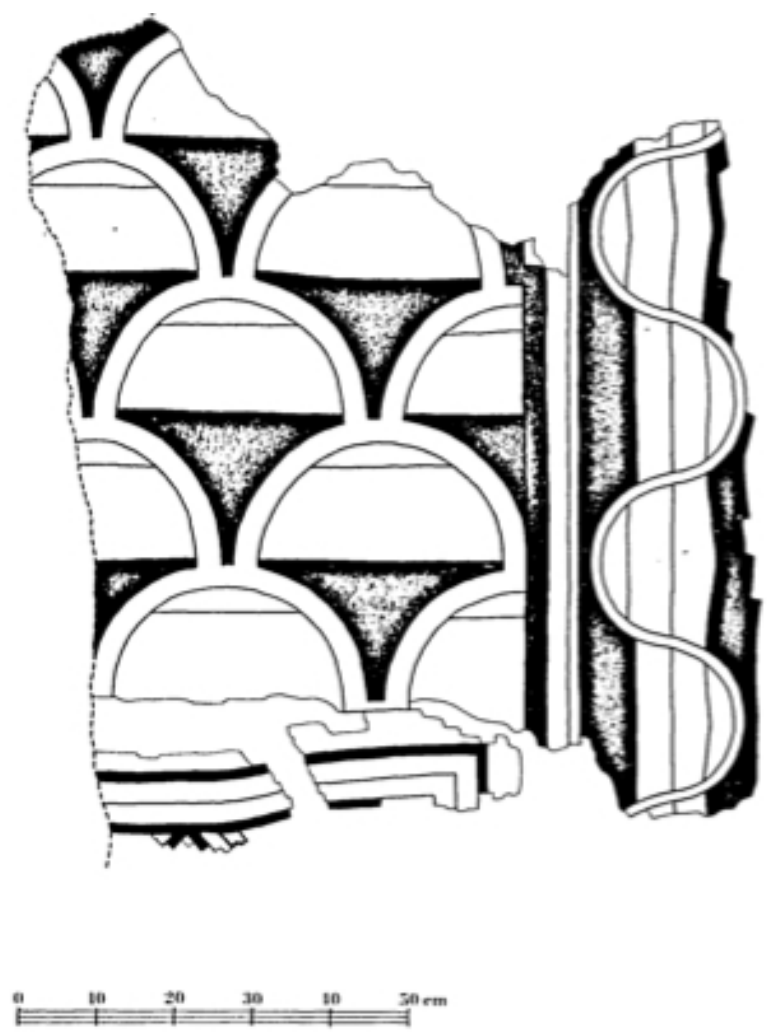

Figura 14 Pavimento 15, Basilica di San Saturnino (da Mureddu-Stefani 1986) mondo punico, raffigurato sia in placchette ossee, sia in medaglioni bronzei, nelle ceramiche e nelle steli, mentre nei pavimenti la troviamo a Delos (Bruneau, 1972, 71) in Sicilia (Tusa - De Miro, 1983, 107) a Cartagine in un tempio dedicato alla stessa dea Tanit (Mancini, 2012, 65) e a Kerkuan, dove il pavimento presentava diversi simboli raffiguranti la dea (Dalmazzo, 2010, 11). Tutte queste attestazioni hanno un arco cronologico che va dal IV secolo a.C. sino alla prima metà del I secolo a.C. Per quanto riguarda la figura circolare A. M. Bisi ci dice che ha un significato astrale, probabilmente raffigurante il sole (Bisi, 1967, 50). Generalmente questi cementizi sono datati a una generica età repubblicana (Angiolillo, 1981, 107), ma credo che possiamo datarli ad un III-II secolo a.C., in virtù del fatto che il pavimento non subì manomissioni in antico, dunque nessun restauro, e sopratutto per l'affievolirsi del culto della dea Tanit in Sardegna con la conquista romana.

\section{Basilica di San Saturnino: la Necropoli}

Dalle ricerche archeologiche effettuate dal 1979, Letizia Pani Ermini afferma che l'area basilicale fu frequentata sin dal III secolo a.C. Edifici realizzati in conci di calcare teneri e laterizi, sepolture a fossa o alla cappuccina e sarcofagi interrati sono databili in piena età imperiale (Pani Ermini - Spanu, 1992, 7-13). La necropoli fu ampiamente frequentata almeno dal II al IV secolo d.C., sia da cristiani che pagani, anche se successivamente il suo assetto $\mathrm{fu}$ stravolto per far spazio alla realizzazione di nuovi edifici, in alcuni casi demolendo quelli esistenti (Salvi, 2007, 350).

\section{- Pavimento 15}

Si tratta di un frammento decorato da una composizione ortogonale di squame ombreggiate adiacenti, in colori contrastanti, simili ad un'embricatura, in bianco, ocra e nero, il bordo presenta una decorazione a onde con tessere bianche, grigie e ocra (Fig. 14). Questo pannello a ovest presenta una cornice, con molta probabilità una cornice di una tabula andata o una tabula semplice. Confronti calzanti li troviamo a Uppenna in almeno quattro esemplari, tutti datati tra la seconda metà del IV e gli inizi del V (Raynal, 2005, $434 ; 471 ; 473 ; 527)$. Si propende per una datazione alla prima meta del V secolo d.C. 


\section{- Pavimento 16}

Un frammento con molteplici guasti, rimane integra solo la parte inferiore, in tessere bianche, rosse e nere, con un motivo geometrico di difficile lettura.

- Pavimento 17

Frammento esiguo, presenta un bordo a treccia doppia policroma, con tessere in color bianco, nero, giallo, rosso grigio e celeste, apparentemente senza una logica cromatica (Fig. 15). Il bordo inquadra una cornice che reca un'iscrizione della quale restano poche tracce: [sub di]e XIII oppure [in pac]e XIII.

L'epigrafe è di difficile lettura, ma con grande probabilità si tratta di un epitaffio funerario. In Sardegna mosaici simili li ritroviamo a Porto Torres, con una datazione del IV secolo d.C. (Quattrocchi, 2015, 249), propenderei per una datazione simile anche per il nostro esemplare.

\section{Pavimenti Fuori Contesto}

- Pavimento 18

Questo pavimento apparteneva ad un pavimento maggiore che decorava una sala del vecchio Museo Archeologico. Si tratta di un frammento di $1,76 \mathrm{x}$ 0,80 metri, tessere bianche, nere, marrone, ocra, rosa, rosse e gialle di $0,8-1,3 \times 0,8-2 \mathrm{~cm}$. Restaurato in antico con tessere bianche, grigie e rosse di 1 $\mathrm{x} 1,2 \mathrm{~cm}$. Sappiamo che proviene dal quartiere di Stampace (Angiolillo, 1981, 107). Si trova presso il giardino del Rettorato dell'Università degli Studi di Cagliari, in cattivo stato di conservazione. Si hanno due parti nettamente distinte, sebbene combacino alla perfezione. Una treccia a quattro elementi borda una composizione di cerchi secanti, che determinano fiori a quattro petali lanceolati. I fiori hanno petali alternativamente rossi profilati di rosa e bianco. Nei quadrati a lati concavi di risulta vi è una crocetta con petali a squadra, petali che sono marroni e rossi. Uno degli schemi geometrici più comuni nel panorama musivo romano, per almeno sino al IV secolo. In ambiente italico abbiamo attestazioni a Pompei, nella Casa di Championnet (Blake, 1930, 97), ad Ostia nel Sacello del campo di Magna Mater, datato alla prima metà del II secolo d.C. (Becatti, 1961, 172). Fuori dall'Italia si hanno diverse attestazioni in Africa, a Tipasa nella "Maison des Fresques", datato alla prima metà del II secolo d.C. (Baradez, 1961, 162-165), a Sfax, in un triclinio della fine del III secolo d.C. (Fendri, 1963, 11), e infine, anche se di epoca tarda, a Ippona, nella Grande Basilica, della prima metà del V secolo d.C. (Marec, 1958, 85). Oltre agli esempi africani si possono citare l'esemplare in Betica, ad Italica, nella Casa di Nettuno, datato non oltre il II secolo d.C. (Quattrocchi, 2011, 37).

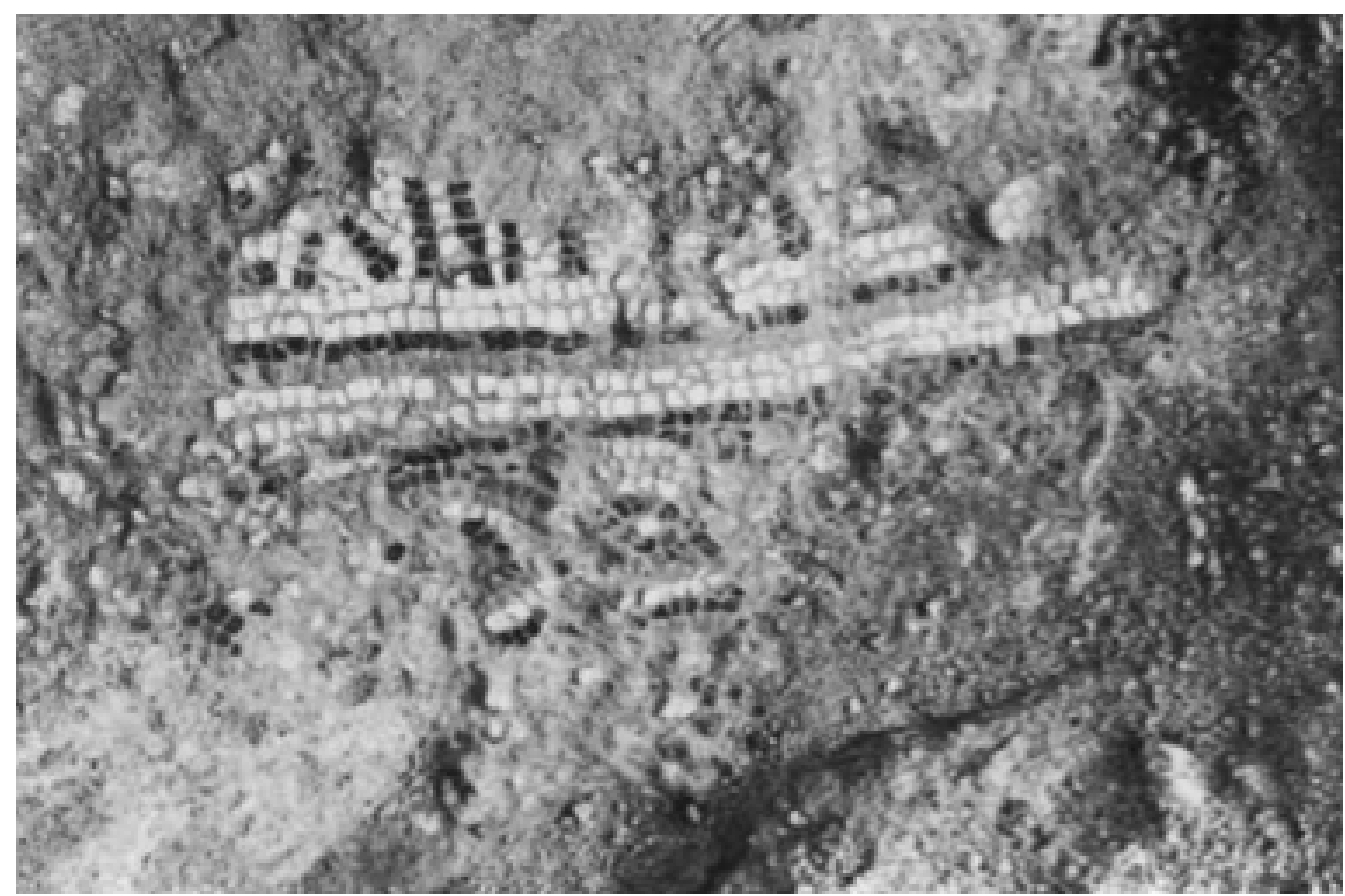

Figura 15 Pavimento 16, iscrizione, Basilica di San Saturnino (da Mureddu-Stefani 1986) 
S. Angiolillo data questo mosaico a un III secolo inoltrato (Angiolillo, 1981, 103), poiché lo ritiene troppo carico di colori. Io opterei per una seconda metà del II secolo, poiché proprio l'esecuzione, di matrice africana, risulta più snella e meglio organizzata, e non sciatta come due esemplari del tutto simili ritrovati a Nora (Angiolillo, 1981, 33; 45) dunque si avvicina di più al modello africano di inizi II secolo d.C.

\section{- Pavimento 19}

Restano solo due frammenti a) di 0,17 x 0,13 metri con tessere bianche, grigie, nere, verdi, marroni, gialle, e rosa di 0,1-0,5 di lat, b) di 0,13 x 0,13 metri. Tessere bianche, marroni, brune, gialle, grigie, rosse e verdi di 0,1-0,5 cm di lato. Conservati presso il Museo Archeologico Nazionale di Cagliari senza numero di inventario, in mediocre stato di conservazione.

Frammento a): Il fondo grigio chiaro presenta un cane in corsa verso la destra, con le zampe posteriori poggianti a terra e le anteriori sollevate. Il contorno del cane è reso da tessere grigie e verdi

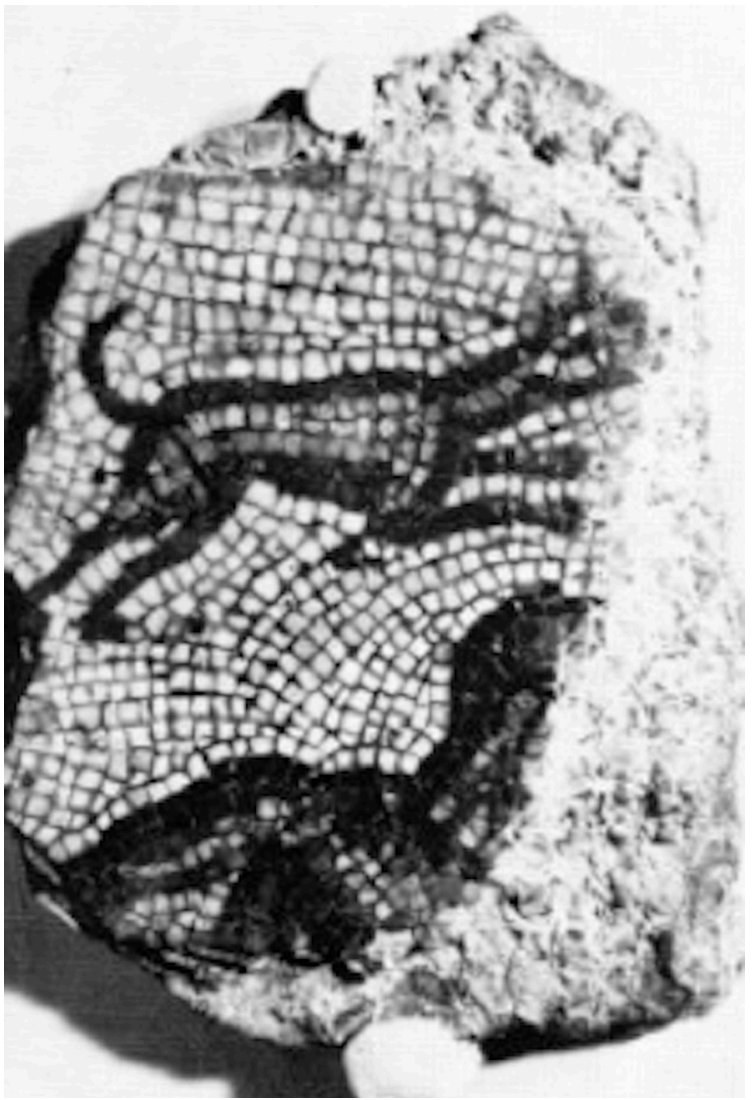

Figura 16 Pavimento 19, cane in corsa (da Angiolillo 1981) scure, il corpo è uniformemente grigio, gli occhi sono evidenziati da tessere bianche. Sotto l'animale vi è un uomo, nudo, del quale si intravede il torso e la barba, resa in tessere marroni (Fig. 16).

Frammento b): Della figura resta solo il collo e il capo di un cervo. I contorni sono in tessere marrone scuro, le corna verdi, mentre il corpo è color bruno e giallo, senza nessuna resa anatomica col chiaroscuro (Fig. 17).

Questi due frammenti si riferiscono ad un pavimento unico, e hanno corpi segnati da forti contorni che ne annullano l'anatomia. I confronti diretti si hanno a Zliten, nel Mosaico dei Gladiatori, del II secolo d.C. (Aurigemma, 1960, 115-116), che offre spunti per le figure singole nella sua composizione, come ad esempio il cacciatore immediatamente sotto il cane. La frammentarietà del pavimento non permette ulteriori confronti, tranne per il cane, che ha un confronto preciso con un mosaico conservato alla Centrale Montemartini, proveniente dagli Horti Liciniani, che però è datato agli inizi del IV secolo d.C., mentre per questi pavimenti sembra più corretta una datazione del II secolo d.C., perché ha precisi confronti col citato Mosaico dei Gladiatori.

\section{- Pavimento 20}

Questo pavimento fu scoperto nel quartiere di Stampace nel 1762 (Angiolillo, 1981, 99) e

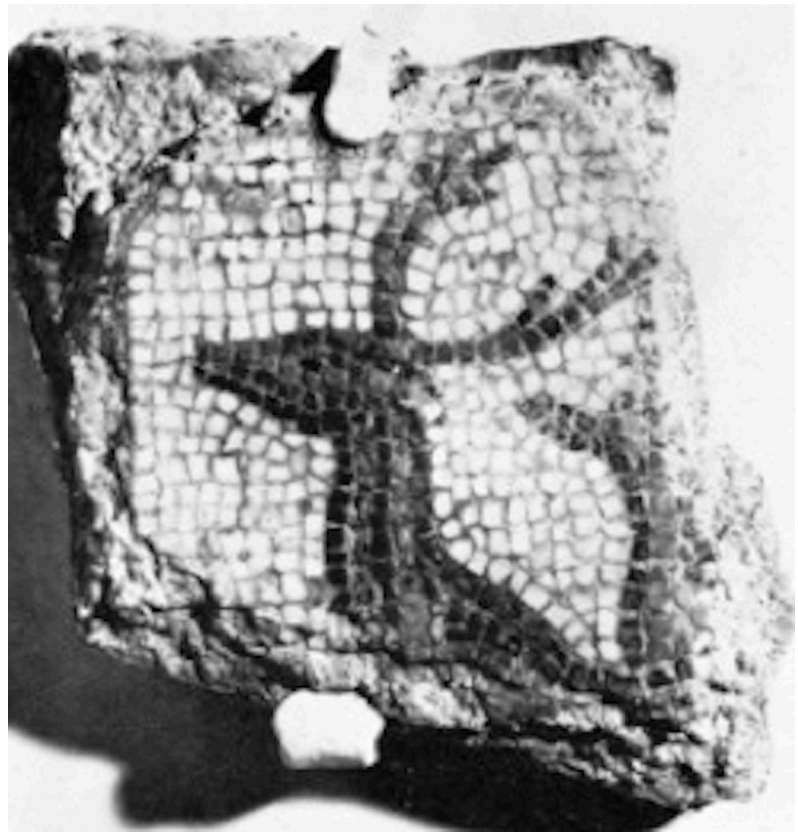

Figura 17 Pavimento 18, cervo (da Angiolillo 1981) 
successivamente trasportato a Torino; durante il viaggio il pavimento rimase gravemente danneggiato, e ad oggi si conservano solo quattro pannelli, di cui uno seriamente lacunoso. I frammenti sono: a) di 2,59 x 1,63 metri; $b$ ) di 1,03 x 0,88 metri; c) di $0,96 \times 0,92$ metri. Le tessere utilizzate sono di colore bianco, nero, grigio, ocra, rosso, marrone e verdi, e hanno dimensioni di 0,5-1 di lato. Si trova conservato presso il Museo Archeologico Nazione di Torino, in ottimo stato di conservazione. Originariamente il pavimento era molto più grande, ne rimane una testimonianza in un disegno del pittore Domenico Colombino. Il campo presentava la figura di Orfeo con la lira, alla sua destra vi era una volpe, e dietro queste due figure due alberi, in uno dei rami di questi vegetali era posato un corvo. Attorno a questo gruppo ruotavano altre figure di animali lungo tutti i bordi, col dorso rivolto a Orfeo. Il pannello $a$ (Fig.18) è interamente occupato dalla figura di Orfeo, il quale risulta a torso nudo, seduto su una roccia, con un mantello rosso che gli passa dietro le spalle, con la mano sinistra suona la lira a sette corde, mentre con la destra tiene il plettro. Sul capo ha il classico berretto frigio rosso, dal quale sbucano scomposte ciocche di capelli. La figura è resa con grande accuratezza, infatti le masse muscolari sono evidenziate da frequenti passaggi di luci e di ombra. Il volto del personaggio è dotato di grossi occhi tondeggianti, sopracciglia unite, un grosso naso e una bocca carnosa. Il pannello $b$ presenta solo la metà di un cavallo, la parte anteriore per esattezza. Il cavallo è in corsa verso destra, le zampe sollevate e la criniera mossa. La tecnica di realizzazione dell'animale presenta le stesse caratteristiche di Orfeo, ovvero con colori giustapposti: il corpo dell'animale è in tessere bianche, nere, grigie, ocra e verdi. Il pannello $c$ ci restituisce l'immagine di un capriolo, con coda e orecchie corte, zampe con l'unghia fessa, e sta brucando dell'erba di una pianta molto simile al fico d'India. Il capriolo è reso con tessere rosse, nere, ocra e marroni. Dal disegno del Colombino si evince che tutti gli animali rivolgano il dorso verso Orfeo. Questa caratteristica non è da attribuirsi a un errore di interpretazione, ma piuttosto riconduce alla praticità: infatti con uno schema simile tutti gli "spettatori" poteva usufruire di una visione migliore del pavimento. Il panorama romano ha moltissimi esempi di mosaici raffiguranti Orfeo: a Volubilis, in un pavimento circolare del III secolo

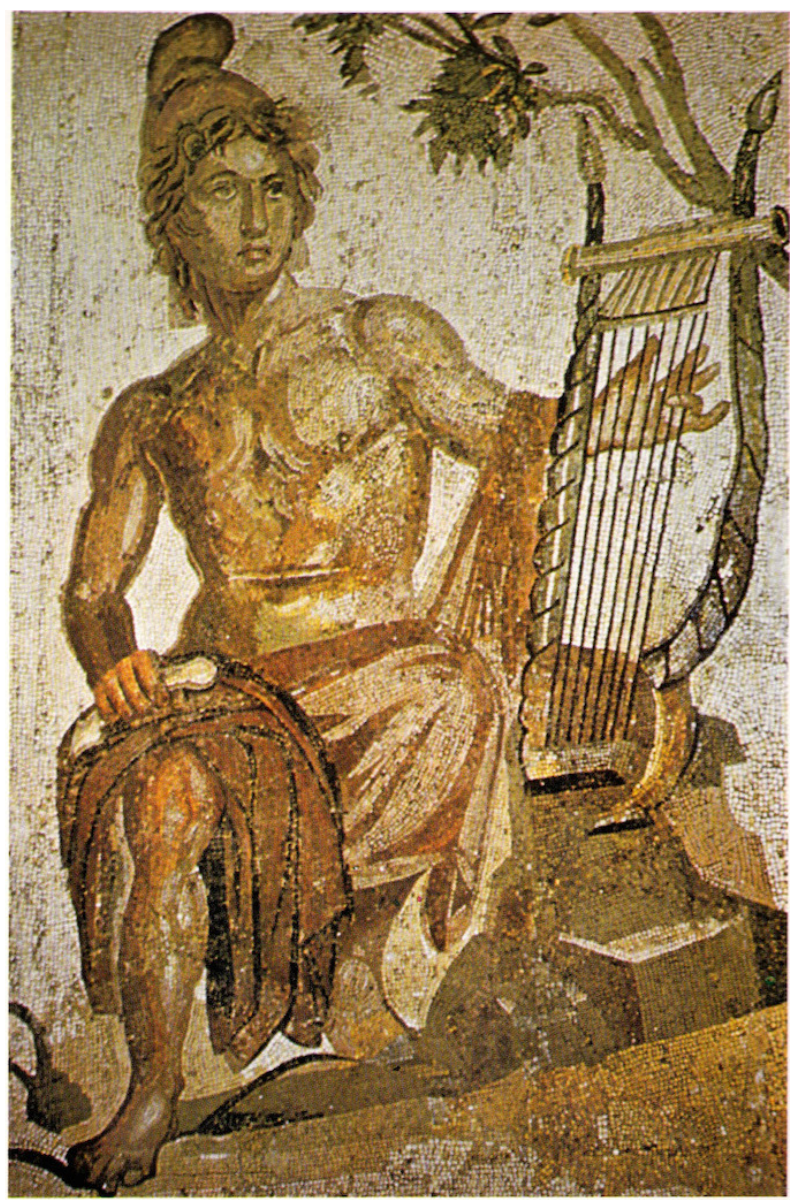

Figura 18 Pavimento 20, Orfeo (da Angiolillo 1981)

d.C., nel quale vediamo Orfeo attorniato in cerchio dagli animali (San Nicolás Pedraz, 2002, 1076), a Badajoz, in Spagna, ritroviamo uno schema simile, datato al IV secolo (Blázquez Martínez, 2008, 1402), all'età di Giustiziano risale un pavimento di Gerusalemme (Campanella, 2006, 1222). I confronti più stringenti però li abbiamo con tre pavimenti: col mosaico di Rottweil datato alla metà del III secolo d.C. (Jesnick, 1997, 136), in Croazia, a Salona nel pavimento del III secolo d.C. (Jesnick, 1997, 138) e infine a Mileto con l'esemplare del 225 d.C. (Jesnick, 1997, 139). La composizione nel suo insieme è curata, le tessere sono minute e ben posizionate, siamo di fronte a una bottega con una consolidata esperienza nelle tematiche figurate, di sicura provenienza africana per la maestria dei passaggi di colore, rapidi e dettagliati. I confronti ci indicano una datazione che non vada oltre la seconda metà del III secolo d.C. 


\section{Conclusioni}

Cagliari doveva essere una città molto importante per la Sardegna Romana, sebbene gli esili reperti non permettano di capirne al meglio l'importanza. Di tutto rispetto è però la sua cultura musiva: dai cementizi del III secolo a.C. fino ai mosaici funerari del V secolo d.C., Cagliari racchiude tante realtà musive, molto differenti tra di loro, che ci fanno comprendere quanti scambi di merci, maestranze e persone dovevano avvenire nel suo porto.
A Cagliari è altresì evidente il passaggio che si ha tra maestranze italiche e maestranze africane, giunte nell'Isola almeno a partire dal III secolo d.C., e che porteranno schemi geometrici e figurati del tutto innovativi per il panorama musivo sardo. A fianco di botteghe locali, identificabili nei pavimenti $1 ; 9 ; 12-14$, abbiamo tutta una serie di mosaici di matrice chiaramente africana, con punte d'eccellenza come il pavimento 20. Da segnalare una grande percentuale (Fig. 19-20), rispetto alla media isolana (Angiolillo, 1981) di mosaici figurati, in un'area dove regnava il mosaico geometrico.

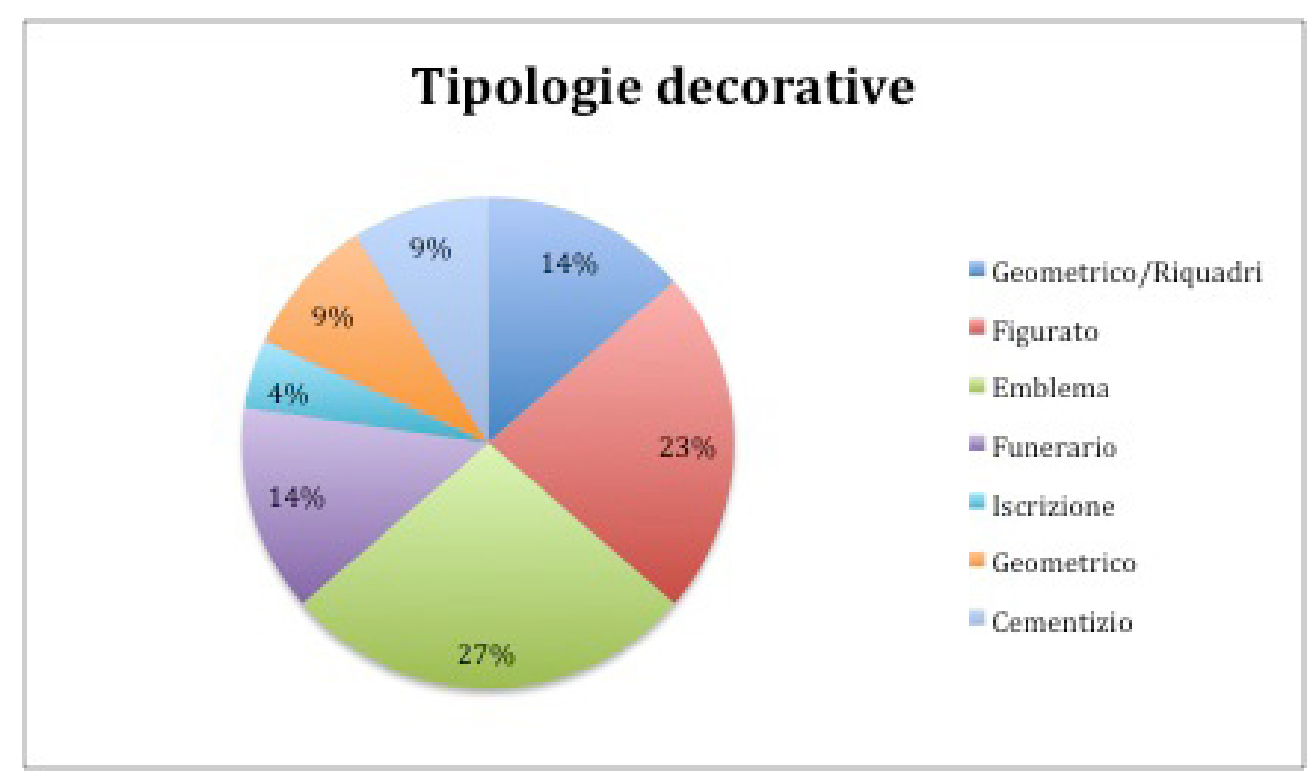

Figura 19 Tipologie decorative nei mosaici di Cagliari

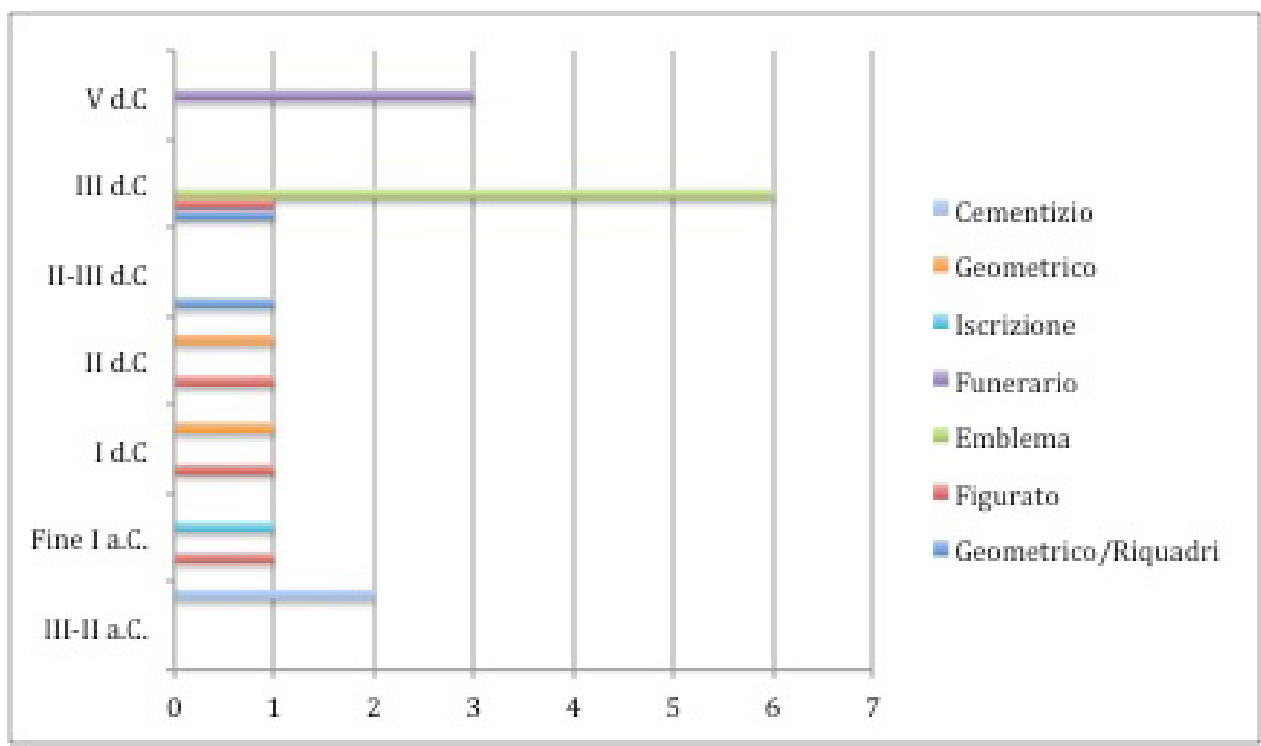

Figura 20 Scansione cronologica e tipologie dei mosaici di Cagliari 
Bibliografia

Angeletti, G. (1997), Il mosaico del Leone in Palazzo Savini, Teramo.

Angiolillo, S. (1981), Sardinia. Mosaici Antichi in Italia, Roma.

Angiolillo, S. (1986), "Modelli africani nella Sardegna di età romana: il mosaico di Santa Filitica a Sorso", L'Africa Romana Atti del IV Convegno di Studi, (Mastino, A.), Sassari, 607608.

Angiolillo, S. (1987), L'arte della Sardegna romana, Milano.

Aurigemma, S. (1960), L'Italia in Africa. Le scoperte archeologiche (a. 1911-a.1945). Tripolitana I: I mosaici, Roma, 50.

Baradez, J. (1961), La Maison des Fresques et les voies la limitant, Tripoli.

Becatti, G. (1961), Gli scavi di Ostia IV. Mosaici e pavimenti marmorei, Roma.

Bisi, A. M. (1967), Le stele puniche, Roma.

Blake, M. E. (1930), The Pavements of the Roman Buildings of the Republic and Early Empire, Roma.

Blazquez Martínez, J. M. (2006), "Mosaicos de Mauretania Tingitana y de Hispania. Temas", L'Africa Romana Atti del XVI Convegno di Studio, (Akerraz, A., Ruggeri, P., Siraj, A. y Vismara, C.), Sassari, 1396-1409.

Blazquez Martínez, J. M. (2008), "La riqueza de Africa a través de los mosaicos”, L'Africa Romana Atti del XVII Convegno di Studi, (Gonzalez, J., Ruggeri, P., Vismara, C. y Zucca, R.), Sassari, 1402.

Bruneau, P. (1972), Dèlos XXIV. Les Mosaïques, Parigi, 72, 102-104.

Campanella, L. (2006), "La circolazione degli animali esotici nell'antichità: il contributo dei vettori fenici e punici”, L'Africa Romana Atti del XVI Convegno di Studio, (Akerraz, A., Ruggeri, P., Siraj, A. y Vismara, C.), Sassari, 1222.

Carandini, A. (1967), La Villa di Piazza Armerina, la circolazione della cultura figurativa africana nel tardo impero ed altre precisazioni, Roma.

Colavitti, A.M. y Deplano, G. (2002), "Evoluzione della forma urbana della città di Carales nel contesto morfologico-ambientale e delle relazioni economico-culturali dell'area mediterranea", Atti del XIV Convegno di Studi sull'Africa romana, (Khanoussi, M.,
Ruggeri, P. y Vismara, C.), Sassari, 1119-1120.

Conti, C. (2012), Claterna. Nuove Scoperte, Roma.

Dalmazzo, M. (2010), Kerkuan e Tanit, Milano.

De Marinis, G. (2009), Pavimenti della Villa del Mito, Sant'Angelo di Vado, Roma.

Dunbabin, K.M.D. (1978), The Mosaics of North Africa, Oxford.

Fendri, M. (1963), "Evolution chronologique et stylistique d'une ensemble de mosaïques dans une station thermaleà Djbel oust", La mosaïque Greco-Romaine. Colloques internationaux du Centre International de la Recherche Scientifique, Parigi, 11.

Foucher, L. (1961), Découvertes archéologiques à Thysdrus en 1960, Tunisi.

Foucher, L. (1963), La Maison de la Procession dionysique à El Djem, Parigi.

Guidi, G. (1935), Orfeo, Liber Pater e Oceano in mosaici della Tripolitana, Roma.

Hanoune, R. (1969), Inventaire des mosaïque de Bulla Regia, Roma.

Kaimio, J. (1969), The Nominative singular in $-i$ of Latin Gentilicia, Londra.

Jesnick, I. J. (1997), The Image of Orpheus in Roman Mosaic, Oxford.

López Monteagudo, G. (2008), "Las riquezas de las aguas en los mosaicos”, L'Africa Romana Atti del XVII Convegno di Studio, (González, J., Ruggeri, P., Vismara, C. y Zucca, R.), Sassari, 2557-2587.

Lugli, G. (1927), "Studi topografici intorno alle antiche ville suburbane: Villa Adriana", Bollettino della Commissione Archeologica Comunale di Roma.

Mancini, L. (2012), Tempio della Dea Tanit a Cartagine. Spunti e riflessioni, Roma.

Marec, E. (1958), Monuments cretiennes d'Hippone, ville espiscopale de Saint Augustine, Parigi.

Mezzolani, A. (2000), "Strutture abitative puniche in Nord Africa: note per un'analisi funzionale", Actas del IV Congreso Internacional de Estudios Fenicios y Pùnicos, Cadiz, 1225.

Neira Jiménez, M. L.,(2006), "Influencias orientales en la musivaria romana de Mauretania Tingitana? A próposito del mosaico denominado del navigium Veneris de Volubilis", L'Africa Romana Atti del XVI Convegno di Studio, (Akerraz, A., Ruggeri, P., Siraj, A. y Vismara, C.) Sassari, 1540. 
Pace, B. (1955), I mosaici di Piazza Armerina, Roma.

Pani Ermini, L. y Spanu, P. G. (1992), Aspetti di archeologia urbana: ricerche nel suburbio orientale di Cagliari, Oristano.

Parlasca, K. (1959), Die römischen Mosaiken in Deutschland, Berlino.

Pernice, E. (1938), Pavimente un figürliche Mosaiken, Berlino.

Puglisi, S. M. (1943), Cagliari. Costruzioni romane con elementi punici nell'antica Karalis, Cagliari.

Quattrocchi, L. (2011), I mosaici romani di Italica, Cagliari.

Quattrocchi, L. (2015), "Maestranze italiche nei pavimenti di Caralis e Turris Libisonis", Saguntum 47, en prensa.

Raynal, D. (2005), Archéologie et histoire de l'Église d'Afrique. UPPENNA II. Mosaïques funéraires et mémoires des martyrs, Tolosa.

Rinaldi, F. (2011a), “Ambienti di rappresentanza”, Atria Longa, 222.

Romanelli, P. (1916), Scavi e scoperte nella città di Tripoli, Roma.

Salvi, D. (2007), "San Saturnino. Progetti di variante", Ricerche a confronto 2006: giornate di studio di archeologia e storia dell'arte (Angiolillo, S., Giuman, M., Pasolini, A.), Cagliari, 350.

San Nicolas Pedraz, M.P. (2002), "Historiografia de la musivaria romana de Mauretania Tingitana", L'Africa Romana Atti del XIII Convegno di Studi, (Khanoussi, M., Ruggeri, P. y Vismara, C.), Sassari, 1076.

Taramelli, A. (1909), Resti di edificio termale scoperti in regione Bonaria, in fondo del Sig. G.B., Ravenna.

Teatini, A. (2007), "Gli scavi recenti nelle terme di Uchi Maius: i mosaici”, Uchi Maius 3: $i$ frantoni: miscellanea, (Vismara, C.), Sassari, 647-648.

Tusa, V. y De Miro, E. (1983), Sicilia Occidentale, Roma.

Vattioni, F.(1979), Nomi e lingue di alcuni popoli semitici, Roma.

Vismara, C. (1984), "I rapporti commerciali tra l'Africa e la Corsica nel VI secolo d.C. i materiali di Castellu", L'Africa Romana Atti del I Convegno di Studio, (Mastino, A.), Sassari, 182.
Zehnacker, H. y Hallier, G.,(1964), Les premiers Thermes de Volubilis et la Maison à la Citerne, Parigi.

Zucca, R. (1996), "Inscriptiones latinae liberae rei publicae Africae, Sardiniae et Corsicae", L'Africa Romana Atti del XI Convegno di Studi, (Khanoussi, M., Ruggeri, P. y Vismara, C.), Sassari, 1459-1460.

FONTI ANTICHE

Cesare, De Bellum Civilis V, 130

Pausania, $X, 17,9$

Plinio il Vecchio, Naturalis Historia III, 7, 35

Solino, Collectanea rerum memorabilium IV, 2

ABBREVIAZIONI

$\mathrm{CIL}=$ Corpus Inscriptiorum Latinarum, $\mathrm{X}$, 7587, 7598, 7599, 7603; XIV, 4549, Berlino 1863-. 